\title{
ERRATA.
}

Page 12, lines 16 and 17 , for one hundred read three hundred and for me thousand read six hundred.

Page 17 , line 2 , dele first letter in the line.

Page 168, line 12, page 177, lines 13 and 14, and page 271, line 10, for Lemna trisulca read Spirodela polynhiza.

Page 209 , line 2 of foot-note, after but insert represchts.

Page 256, line 7, and page 266, line 19: snowi n. s. has been shown to be hieroglyphica, o'.

Page 257, insert as line 8 as follows: -ken to the office produced young in ten days. The

Page 272, line 13, for $P$. biguttatus read Pompilus biguttatus.

Page $₫-8$, Plate V., 16, after view insert as follows: $a$, mentum; $b$, labial rudiment; $c$, maxillary palpi; $d$, maxilla; $e$, labrum; $f$, autenna; $g$, eye; $h$, mandible.

Page 286, line 11, drop initial the one line.

Page 386, line $\vec{i}$, for Comstocki read Comstock.

Page 399, line 17, for specimens read specimen.

Page 411 , line 10, for Michaelson read Michcielsen.

Page 441, line 3 from bottom, for 66 read 68 .

Page 445 , line 10 from bottom, for 57 read 58.

Page 466, line 1 frum buttom, for Cypria read Cypris. 
Article VII.-Descriptions of three New Parasitic Hymenoptera from the Illinois River. BY WILLIAM H. AsHMEAD.

\section{FAMILY PROCTOTRYPIDE.}

\section{Phanurus Thomson.}

Phanurus tabanivorus, sp. n. [Fig. 55, 56.]

\%.-Length 1.2 to $1.3 \mathrm{~mm}$. Polished black, impunctate; the head and thorax clothed with a fine sparse pubescence. Head subquadrate, roundly emarginate behind, a little wider than the thorax; eyes oval, faintly pubescent; antennæ 11-jointed, black, if extended backwards. not quite reaching to the apex of thorax, and terminating in a long fusiform 5 -jointed club, the first joint of which is not quite as wide as the second, ob-trapezoidal, twice as wide as long, the second, third, and fourth joints transverse-quadrate, a little wider than long; the fifth or last joint conical and a little narrower than the preceding joint; the scape is about as long as the funicle with the pedicel, the latter obconical; joints of funicle a little narrower than the apex of the pedicel, the first joint scarcely longer than thick, the second and third small, transverse-moniliform.

Thorax subovoid, not twice as long as wide, the mesonotum scarcely longer than wide, the scutellum lunate, polished, without pubescence; wings hyaline, ciliated, the cilia on the anterior and posterior margins long, much shorter at apical margin; tegulæ black; venation brown, the marginal vein a little shorter than the stigmal, the latter only slightly thickened at tip, the postmarginal vein very long, fully two aud a half times as. long as the stigmal; legs fuscous, the trochanters, knees, tips of tibiæ and tarsi honey-yellow or testaceous. Abdomen elongate, pointed-fusiforn, about twice as long. 
as the head and thorax united, polished, the first segnent not longer than wide, with an elevation above at base, the second segment the longest, twice as long as wide at apex, the suture between it and the first striated, the third segment hardly half as long as the second, the fourth about two thirds the length of the third, the three following forming a cone of which the fifth is very short, its apical margin with a median sinus, the sixth twice as long as the fifth, the seventh very short, scarcely discernible; sheaths of ovipositor a little prominent.

o.-Length $0.8 \mathrm{~mm}$. Black, but with the head, prosternum, and legs testaceous; the antennæ 12-jointed, brown-black, with all the joints of the flagellum, except the pedicel and the last joint, small, moniliform, joints 3 to 5 increasing in size but smaller than pedicel, joints 6 to the penultimate a little wider than long; abdomen not, or only slightly, longer than the head and thorax united, the genitalia long, exserted, curving downwards.

Hab.-Havana, Ill.

Types in collections of the Illinois State Laboratory of Natural History and in my collection.

Described from $11 \sigma$ and $9 \%$ specimens bred from the eggs of a common horse-fly, Tabanus atratus Fabr.

In $\mathrm{my}$ recent monograph of the $\mathrm{N}$. A. Proctotrypidæ, pp. 140-141, I characterized four species belonging to this genus, none of which, however, are closely allied to the present species. On the contrary, it appears to resemble more closely three European species described under the genus Telenomus, viz.: Telenomus othus Hal., T. Laricis Hal., and T. tabani Mayr.

Dr. Gustar Mayr, in his excellent revision of the European species of Telenomus* did not recognize the validity of Thomson's genus Phanurus.

As defined in my monograph, however, I believe it can be readily separated from Telenomus, although both Haliday and Walker have described Telenomi which

"Ueber die Schlupfwespengattung Telenomus." Verh. d. k. k. zool. bot. Govellsoh. in Wien, 1877 , pp. 697-714. 
should now be relegated to Phanurus; while Thomson, in his definition of Telenomus, has included species that really belong to the genus Hadronotus Förster.

These errors probably induced Dr. Mayr to reject the validity of Phanurus in his work cited above.

It is interesting to note here that Phanumu (Telenomus) tabani Mayr, has habits similar to our own, the species having been bred by Dr. Frederick Brauer from a European horse-fly, Tabanus sp.

Our species, although evidently related, is somewhat larger in the female sex, smoother, with the joints of the antennæ and the segments of the abdomen relatively different, while our male is much smaller, differently colored, and with the antennal joints totally dissimilar.

Dr. Mayr's species approaches nearest to Telenomus laricis Hal., figured in the Entomological Magazine, Vol. III., Plate XIIl., Fig. 2, with which he makes comparison; while our species agrees more nearly with Telenomus othus, Hal., represented on the same plate in Fig. 4.

FAMILY BRACONIDA.

Cuinocentrus Haliday.

Clinocentrus niger, sp. n. [Fig. 14.]

o.-Length 1.85 to $2 \mathrm{~mm}$. Black, subopaque, shagreened, except the apical third of abdomen, which is smooth and shining; mandibles rufous, palpi whitish; antennæ 25-jointed, longer than the body, black, except a small honey-yellow annulus at base of third joint and the apex of the pedicel or second joint; all coxæ and trochanters brownish yellow, the femora reddish and gradually becoming fuscous toward tips; all tibiæ and tarsi black or blackish fuscous; wings subfuliginous, the stigma and veins dark fuscous, almost black.

The head is transverse, shagreened, with only the occipital orbits smooth and shining; the occiput is acutely margined; the metathorax is rugulose, with a median carina; the first and second abdominal segments are shagreened or granulated and opaque, the second 
being the longer; the third segment is scarcely half the length of the second, subopaque and only faintly shagreened; while the following segments, in marked contrast to the preceding, are all short, impunctate, and shining. Hab.-Havana, Ill.

Types in the collections of the Illinois State Labora. tory of Natural History and in my collection.

Described from 10 on specimens taken on floating Lemnaceæ in the Illinois River.

\section{Family IChNeUMONIDA.}

\section{Cremastus Gravenhorst.}

Cremastus hartii, sp. n. [Fig. 13.]

9.-Length $6.5 \mathrm{~mm}$.; ovipositor about half the length of the abdomen; antennæ 30-jointed, scarcely reaching to the middle of abdomen. Brownish vellow or pale ferruginous; head above, the occiput, the antennæ, a large oblong spot on middle mesothoracic lobe anterioriy, the forea at base of scutellum, axillæ, postscutellar band, base of petiole, basal half of second abdominal segment, a small spot on dorsum of fifth and sixth segments, all tarsi and the hind tibiæ, black or dark fuscous; wings hyaline, the stigma brown, the veins blackish; abdomen twice as long as the head and thorax united, compressed from the third segment, the petiole and base of third segment finely longitudinally aciculated and feebly shagreened; the second segment one half longer than the third, the sixth shorter, the seventh still shorter. (The eighth segment has the basal plates of the ovipositor abnormally exserted, giving the tip of abdomen an unnatural appearance.)

o'.-Length $4.5 \mathrm{~mm}$. Agrees with female except in the usual sexual difference and in color, the sixth and seventh abdominal segments having a black spot above, while the anterior tarsi and the base of the middle tarsi are pale.

Hab.-Havana, Ill.; Washington, D. C.; Lexington, Ky. Types $\delta^{7} P$ in the collections of the Illinois State Laboratory of Natural History, and in my collection. 


\section{EXPLANATION OF FIGURES.}

\section{Plate I.}

1. Paraponyx obscuratis, larva, dorsal view.

2. Larval and pupal case.

3. Posterior false leg of larva.

4. Gill filament of larva.

5. Abdominal segment of larva, showing bases of filaments and setæ of piliferous tubercles.

6. Pupa, side view.

\section{Plate II.}

7. Paraponyx obscuralis, posterior extremity of pupa, ventral view.

8. Hydrocampa obliteratis, eggs on leaf, natural size.

9. A few eggs enlarged.

10. Larva, dorsal view.

11. Larva in case, posterior extremity visible.

12. Posterior false leg of larva.

\section{Plate III.}

13. Cremastus hartii Ashm., n. s.

$$
\text { Plate IV. }
$$

14. Clinocentrus niger Ashm., n. s.

Plate V.

15. Bittacomorpha clavipes, larva, side view.

16. Head of larva; right side, dorsal view; left side s. $_{\text {, }}$ ventral view.

17. False foot of larva.

18. Thorax of pupa, just before casting larval skin, cut beneath at middle and spread out, showing growth of respiratory tubes. 
Plate VI.

19. Bittacomorpha clavipes, pupa, dorsal view.

20. Portion of respiratory tube of pupa, enlarged.

21. Spine such as the pupa is covered with.

22. Last segment of male pupa, dorsal view, showing scar left by larval respiratory tube.

23. Erioptera? sp.(a), larva, dorsal view.

24. End of last segment of larva.

25. Ventral view of one side of same.

\section{Plate VII.}

26. Symplecta punctipennis, larva, dorsal view.

27. End of last segment of larva.

28. Pupa, dorsal view.

29. Limnophila luteipennis, larva, dorsal view.

\section{Plate VIII.}

30. Limnophila luteipennis, end of last segment of larva.

31. Pupa, dorsal view.

32. Tipula eluta, larva, dorsal view.

33. End of last segment of larva.

\section{Plate IX.}

34. Tipula eluta, pupa, dorsal view.

35. Tipuln larva (b), dorsal view.

36. End of last segment of larva.

37. Tipula sp. (e), end of last segment of larva.

38. Last two segments of female pupa, side view.

39. Pachyrhina ferminea, end of last segment of larva.

\section{Plate $\mathrm{X}$.}

40. Pachyrhina ferruginea, last two segments of female pupa, side view.

41. Chrysops vittatus, larva, dorsal view.

42. Chrysops cestuans, egg mass on stem of Juncus effusus.

43. Tabanus lineola, pupa, dorsal view.

44. Abdominal spiracle of pupa.

45. Left thoracic spiracle of pupa. 


\section{Plate XI.}

46. Tabanus lineola, end of last segment of pupa.

47. Tabanus stygius, larva, dorsal view.

48. Last segment of larva, side view, showing projecting spine.

49. Tabanus sp. (b), larva, dorsal view.

50. Tabanus atratus, larva, dorsal view.

\section{Plata XII.}

51. Tabanus atratus, head of larva, low side riew; $a$, eye; $b$, antenna; $c$, labrum; $d$, mentum; $e$, mandible; $f$, maxillary palpus; $g$, maxilla.

52. Abdominal spiracle of pupa.

53. Left thoracic spiracle of pupa.

54. End of last segment of pupa.

\section{Plate XIII.}

55. Tabanus atratus, egg mass and single egg, latter containing the parasite, both enlarged.

56. Phanurus tabanivorus Ashm., n. s., egg parasite of Tabanus atratus.

\section{Plate XIV.}

57. Stratiomyia norma, larva, dorsal view; and last three segments, ventral view.

58. Odontomyia cincta, egg mass, with all except bottom layer removed from the lower portion, also single egg, both enlarged.

59. Larva, dorsal view, and last three segments, ventral view.

60. Odontomyia vertebrata, larva, dorsal view, and last three segments, ventral view.

\section{Plate XV.}

Examples of breeding-cage and semicircular dip-net in use at the Station. 
PLATE I.
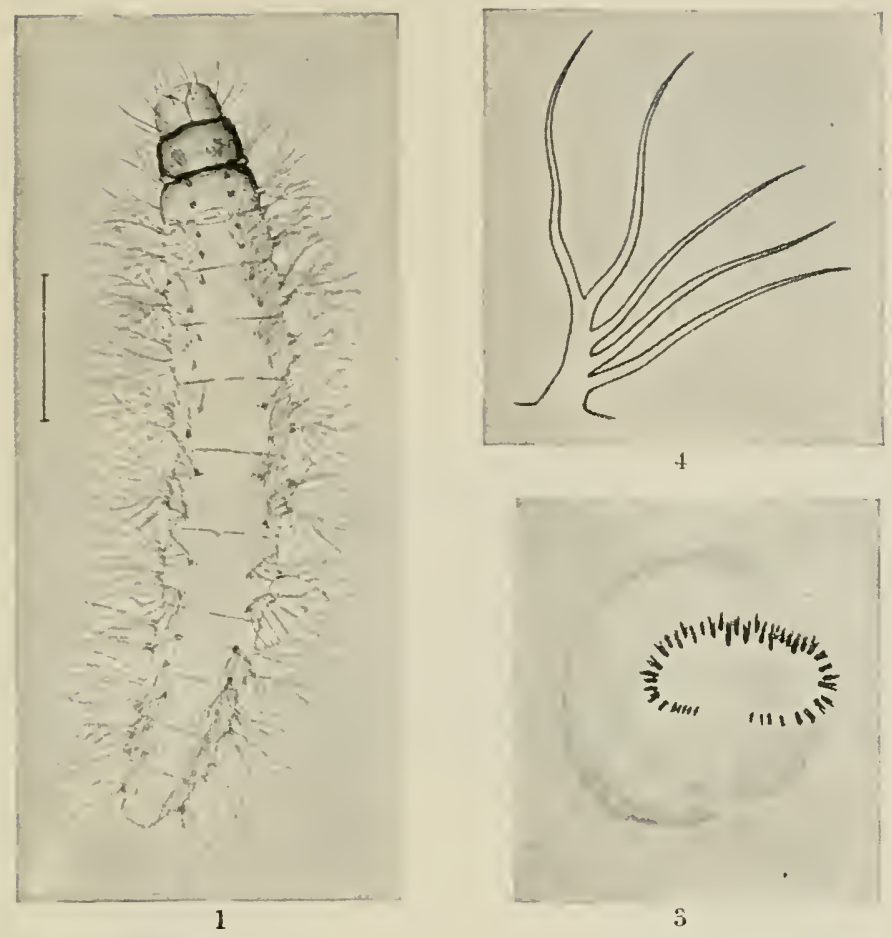

S
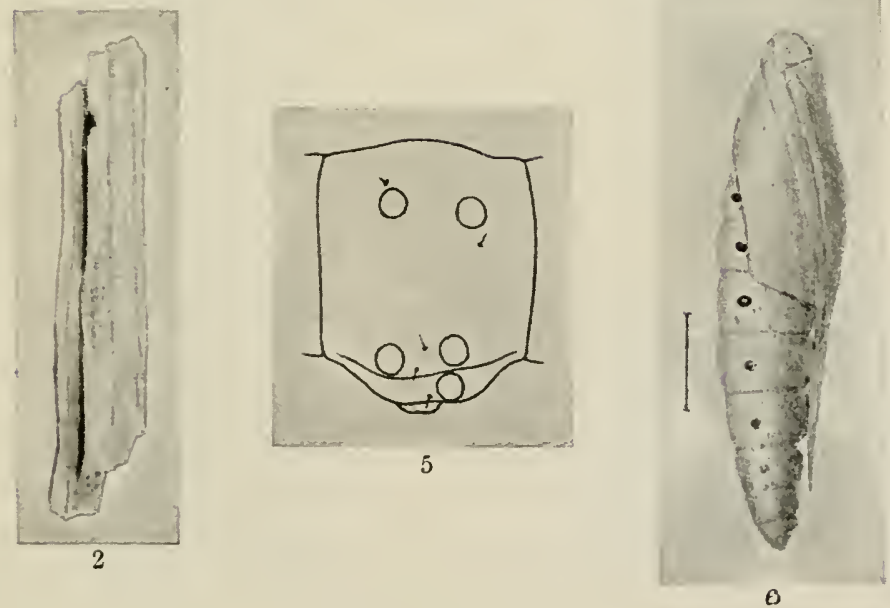


\section{Plate II.}

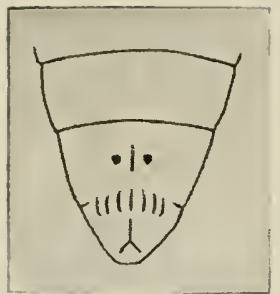

7

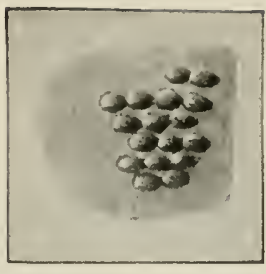

9

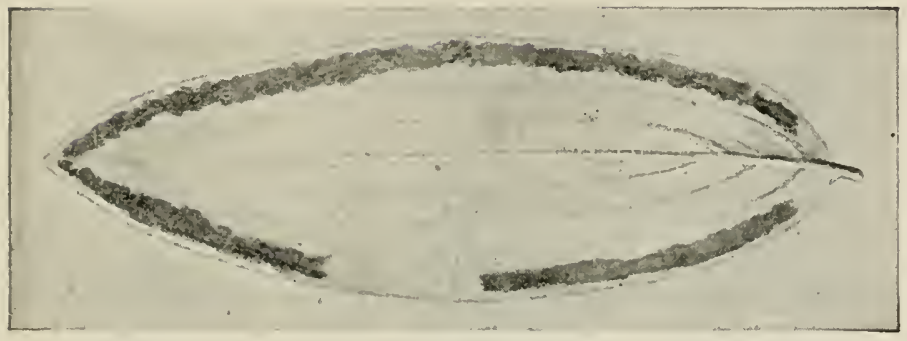

8
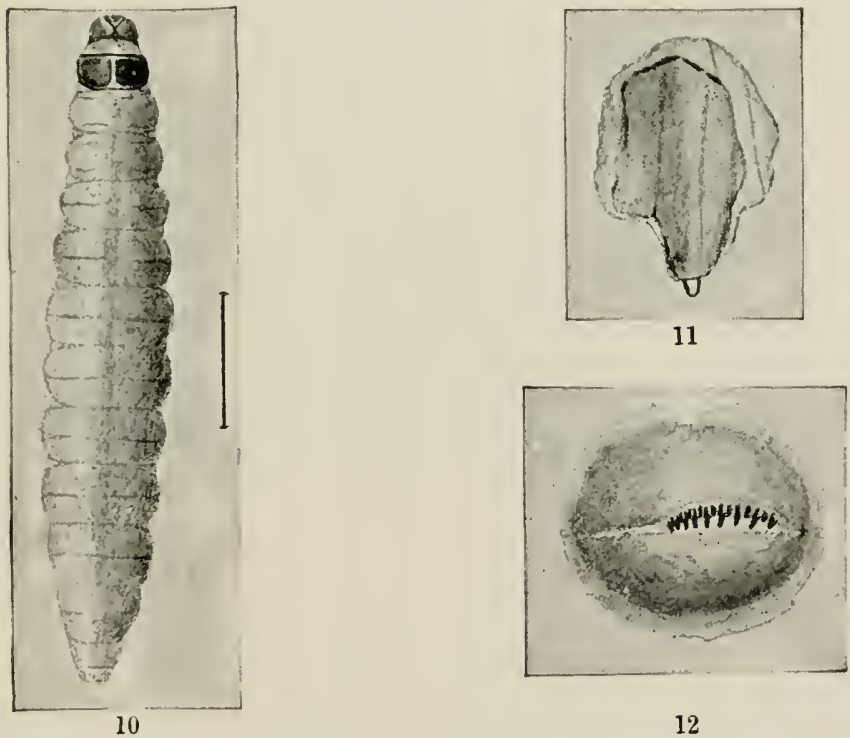
Plate III.

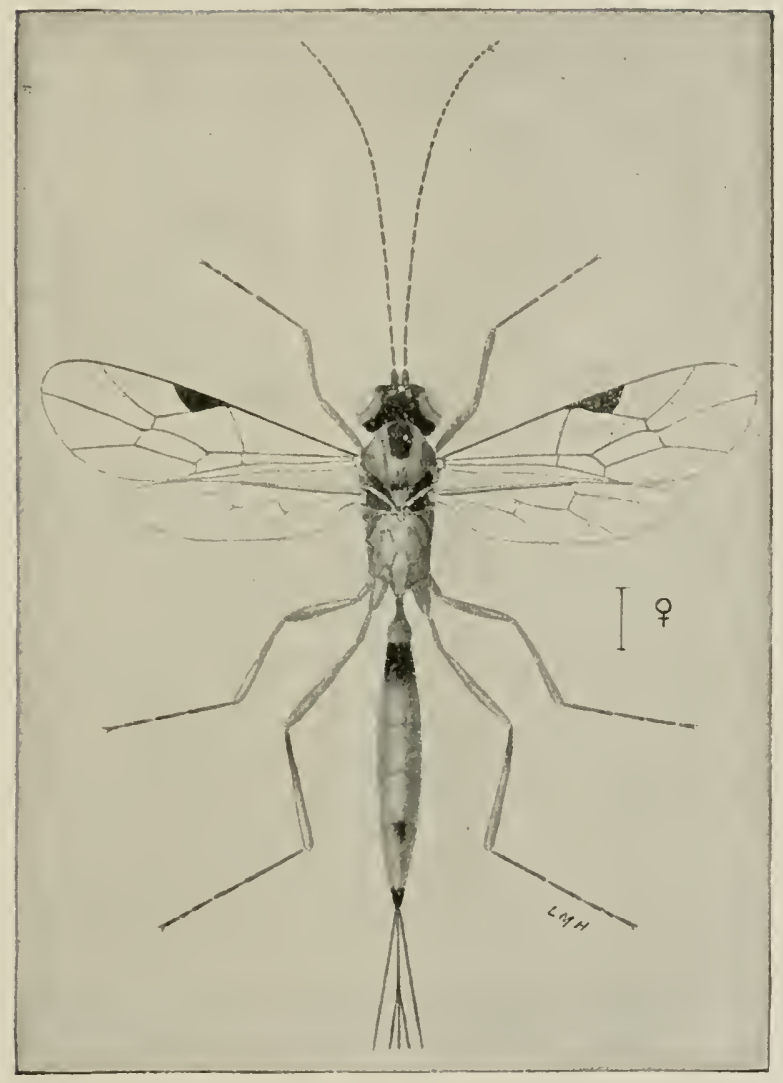


Plate IV.

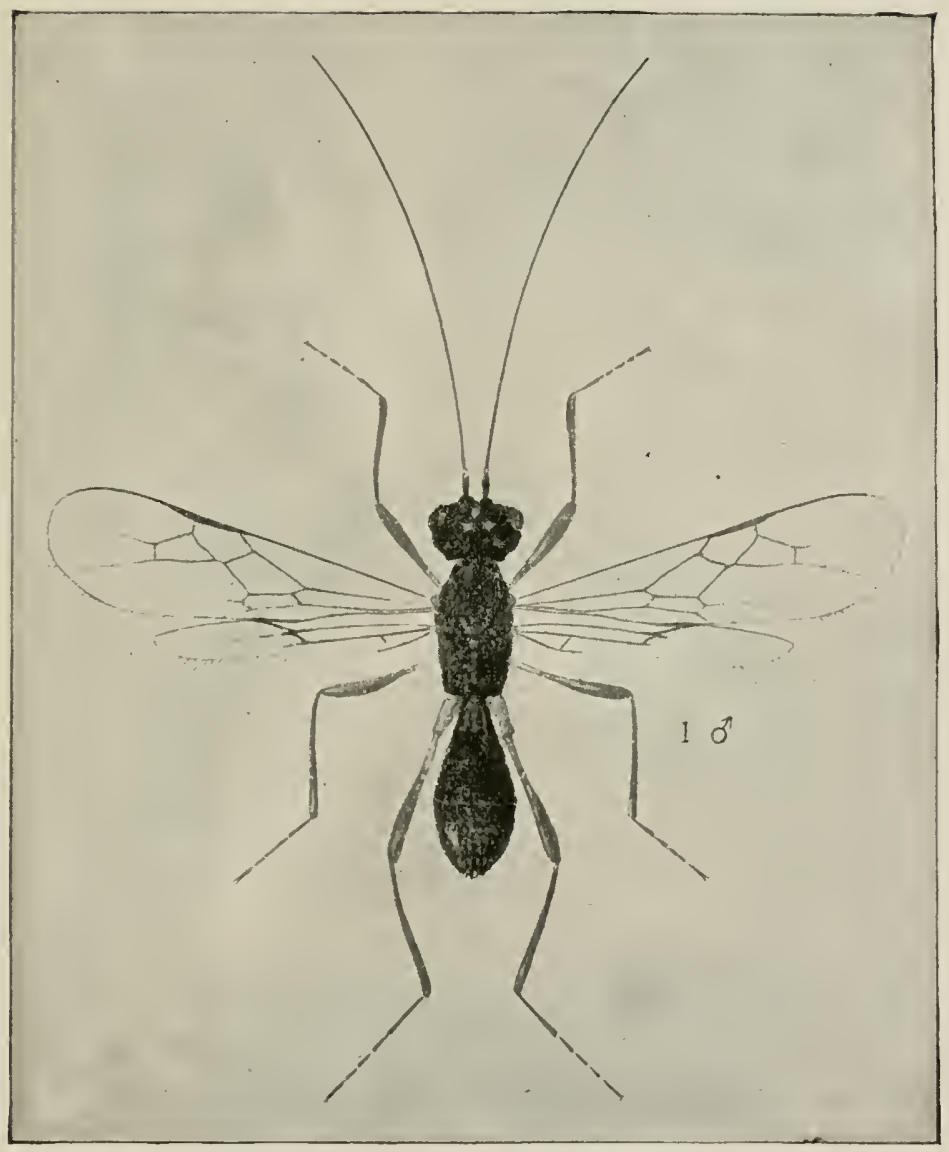

14 
Plate V.
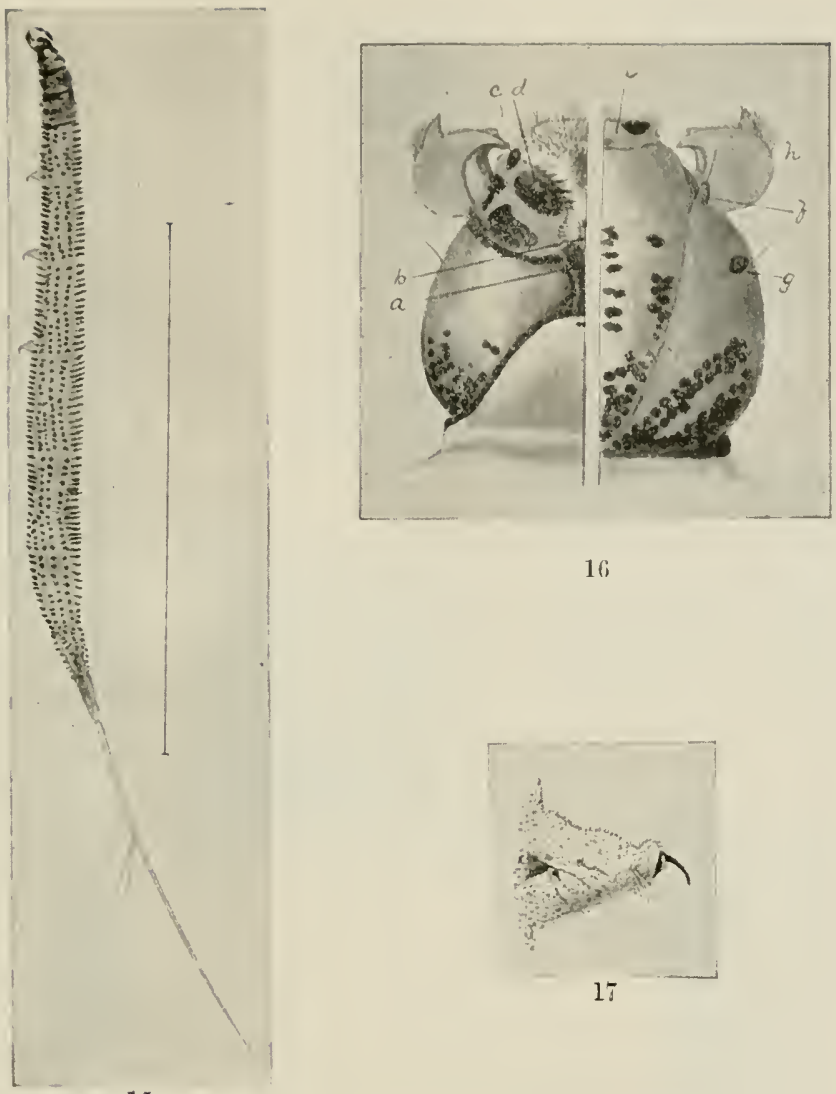

16

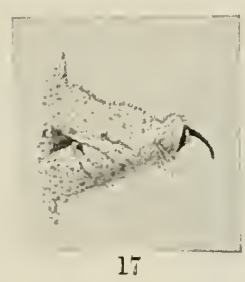

15

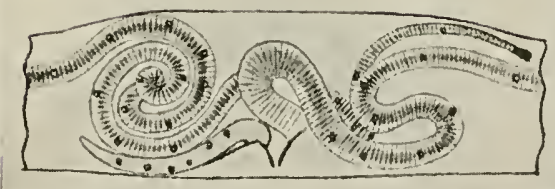

18 
Plate VI.
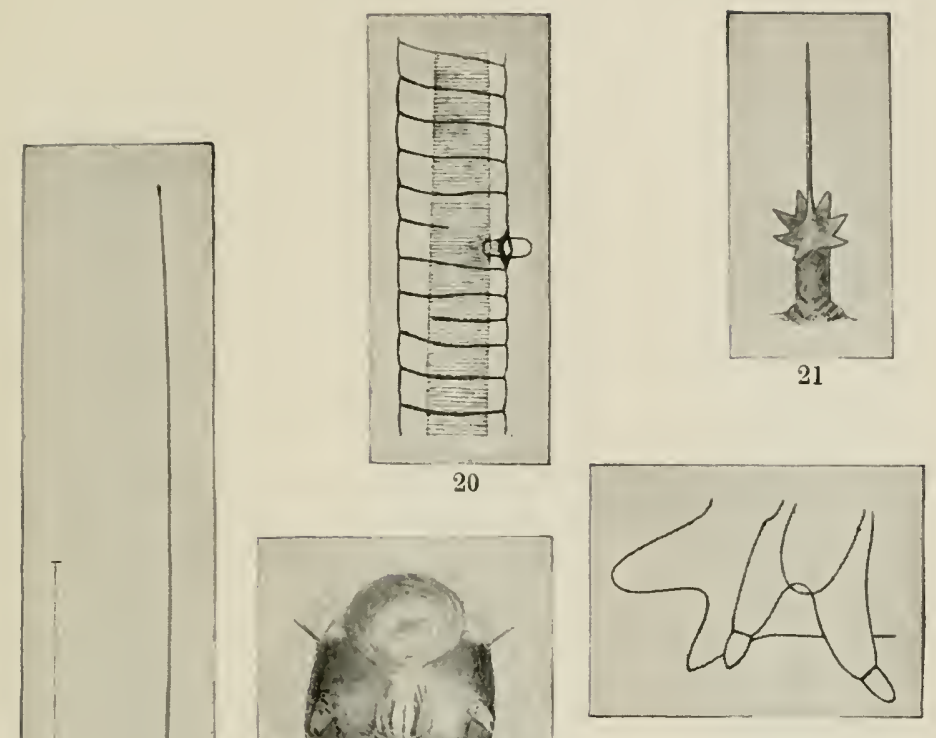

25

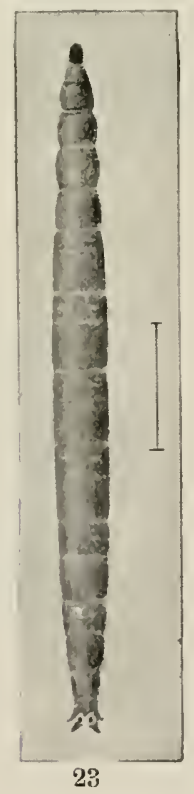

24

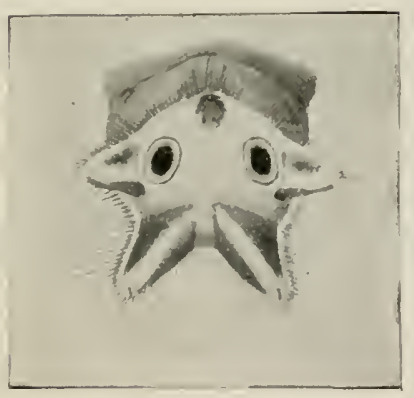

23 
Plate Vil.
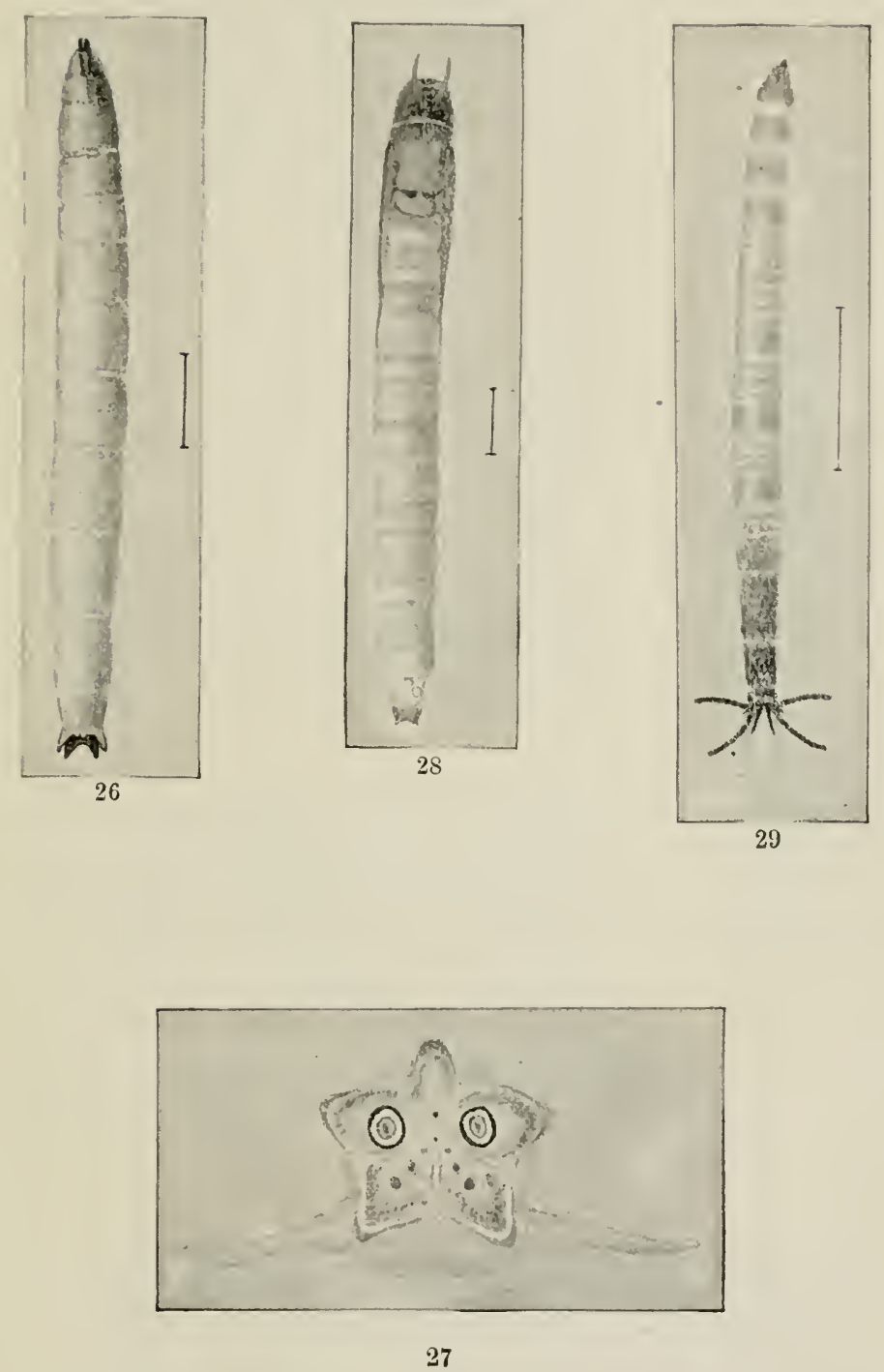


\section{Plate VIII.}
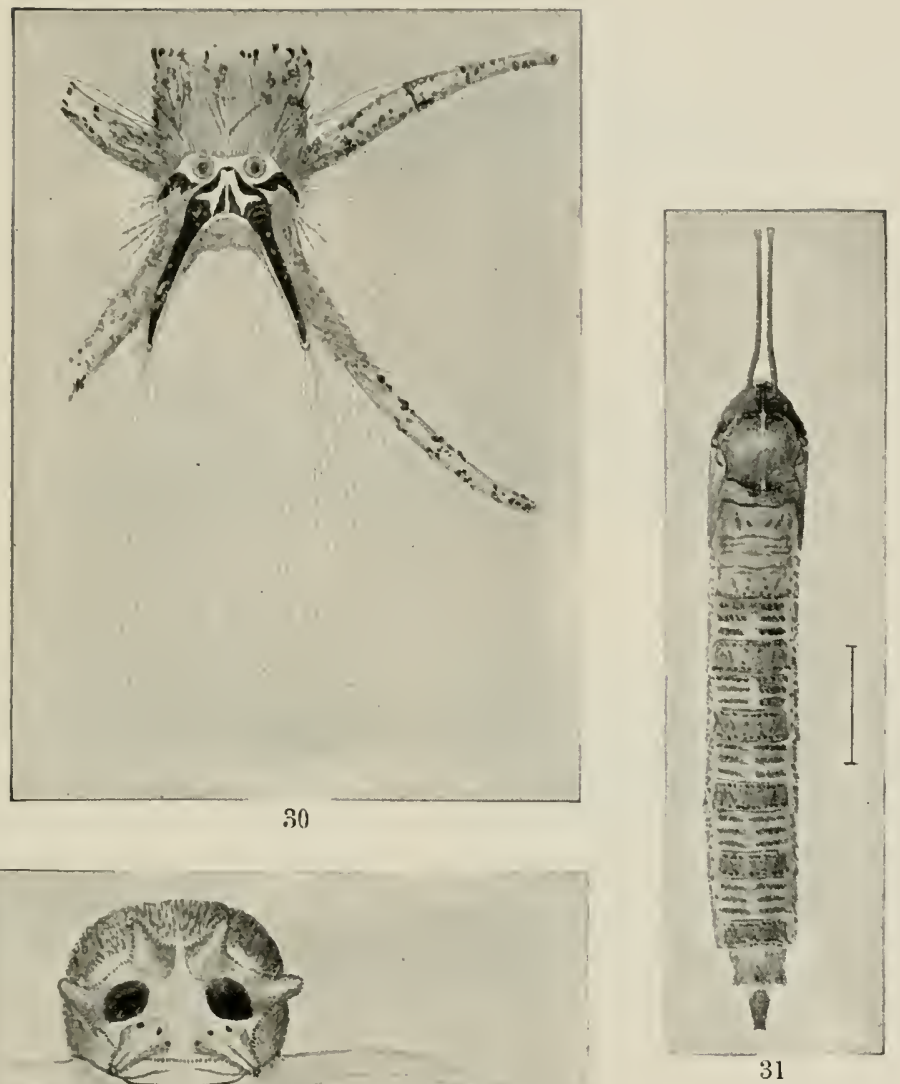

33

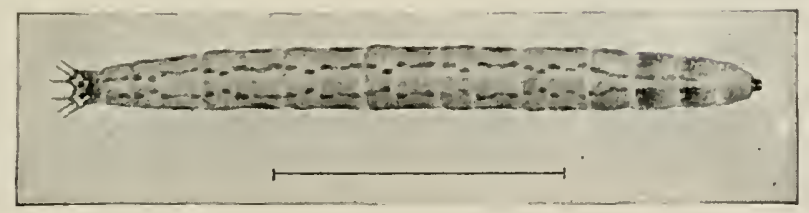


PLATE IX.

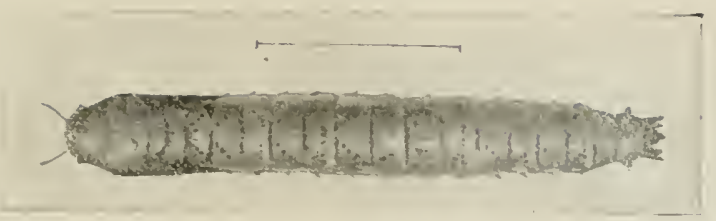

34

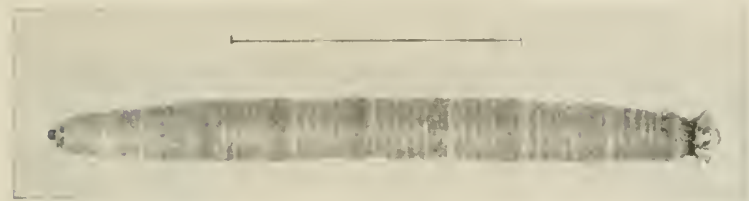

35

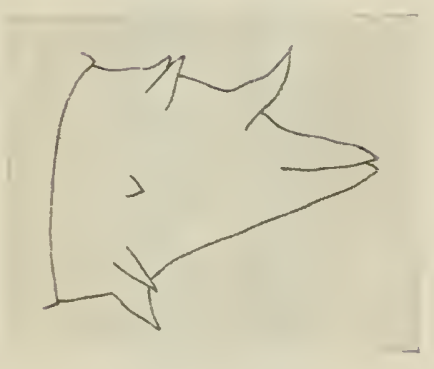

is

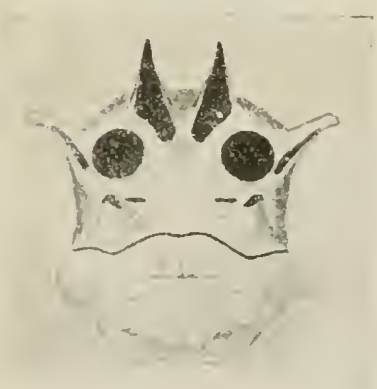

37

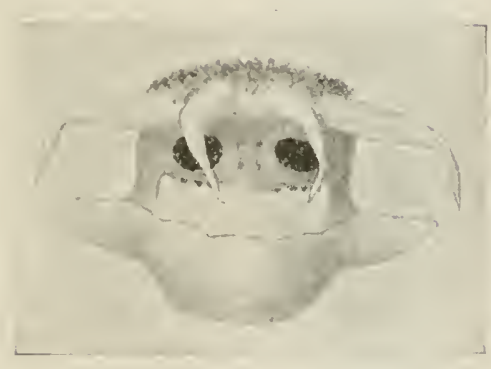

36

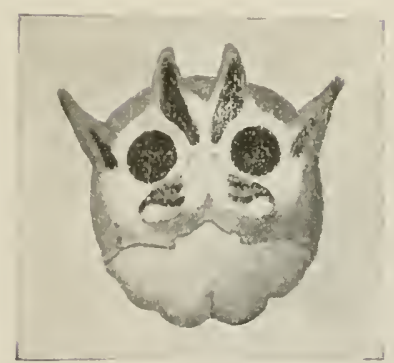

39 


\section{Plate X.}

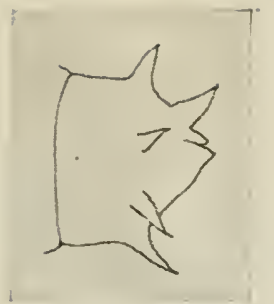

40

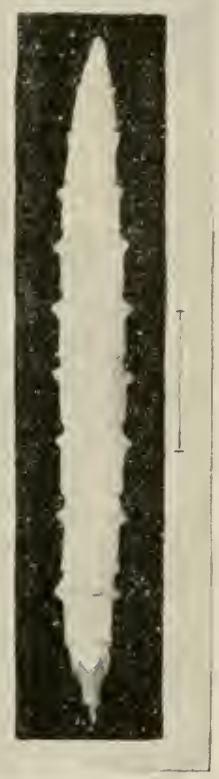

41

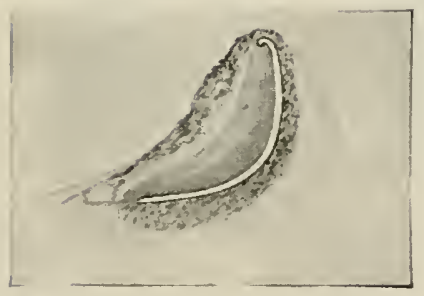

45

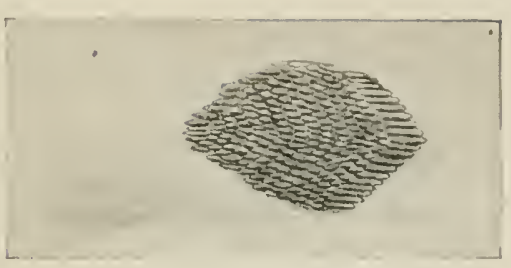

42

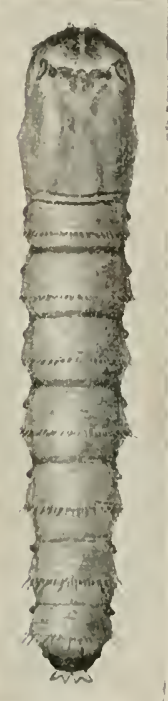

43

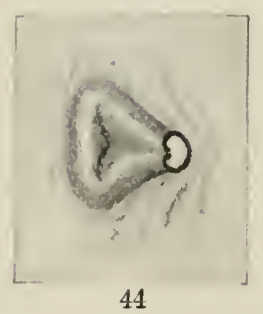


Plate di.

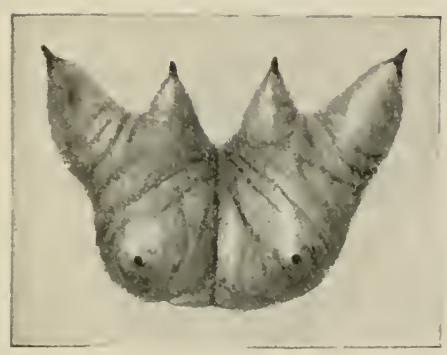

46

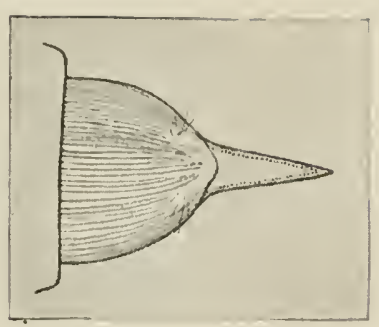

48

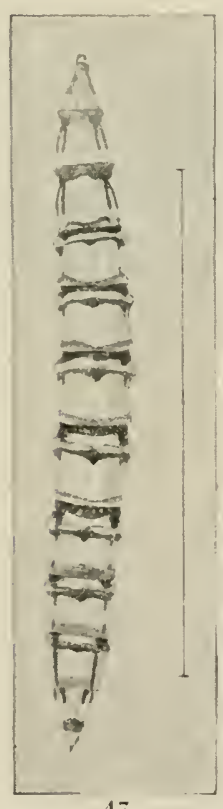

47
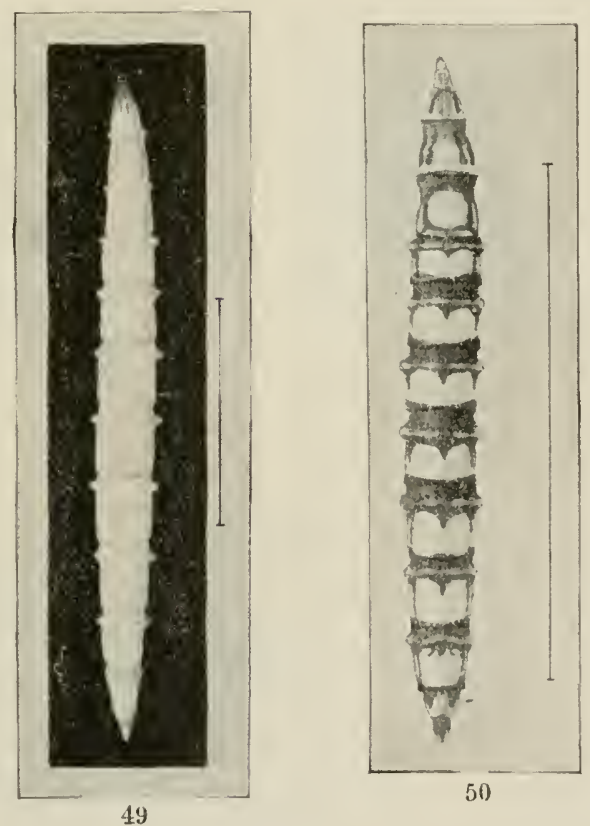
Plate Xil.

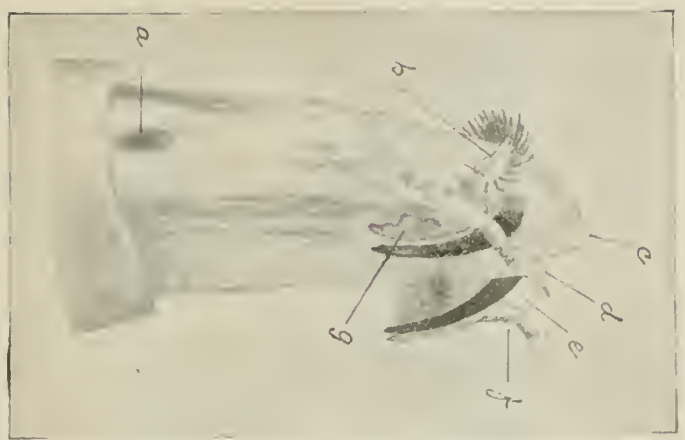

51

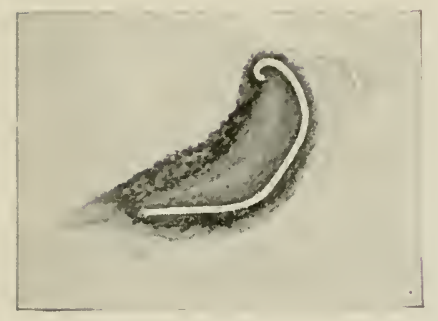

53

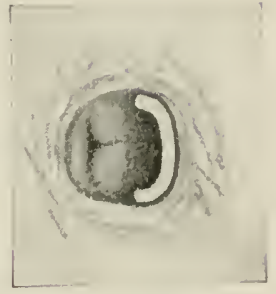

52

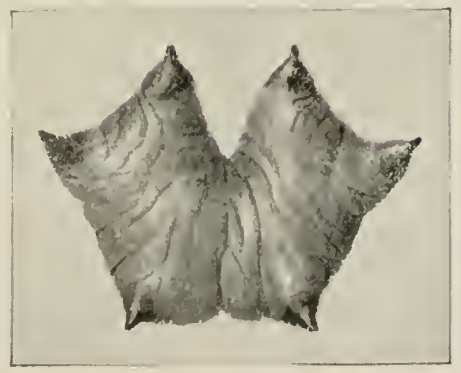

54 
PLATE XIII.

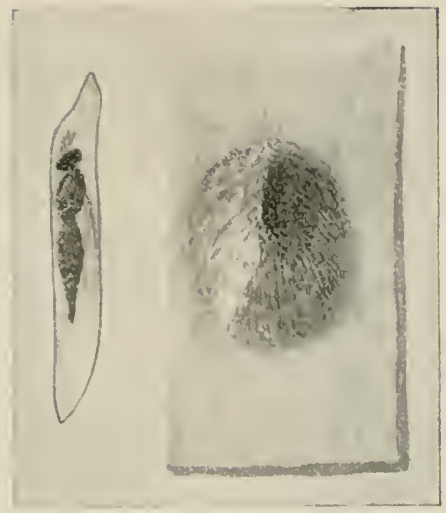

55

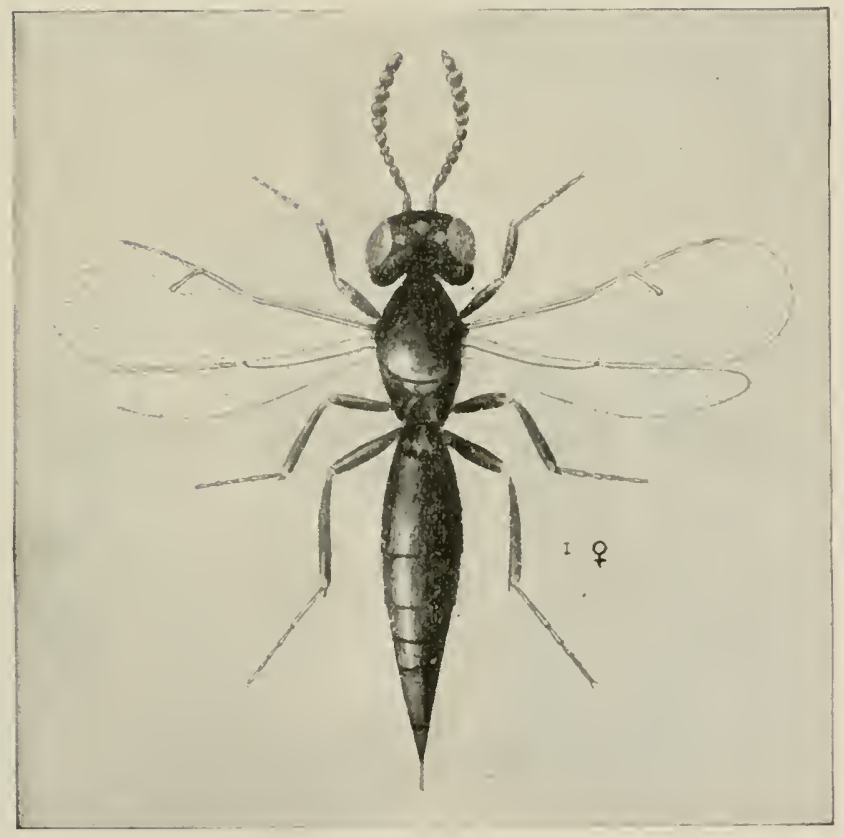




\section{Piate dil.}

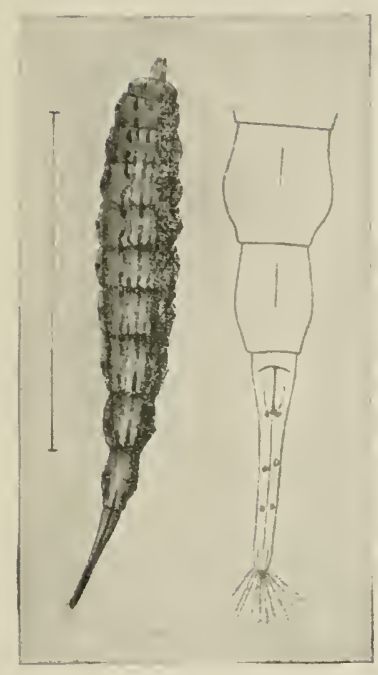

57

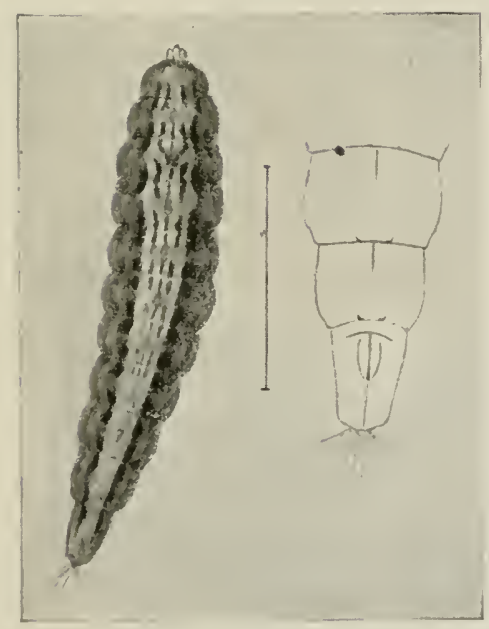

59

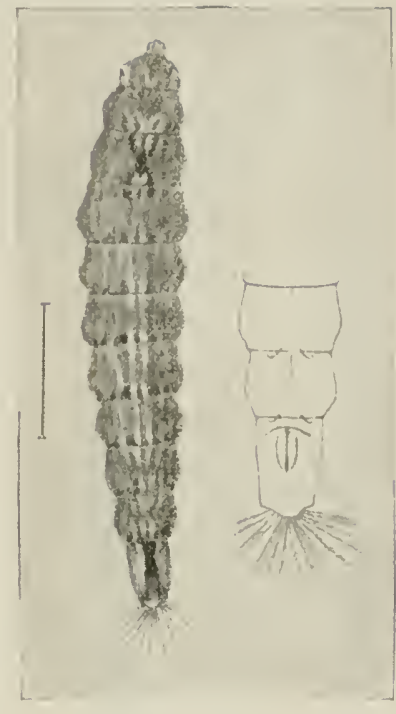

60

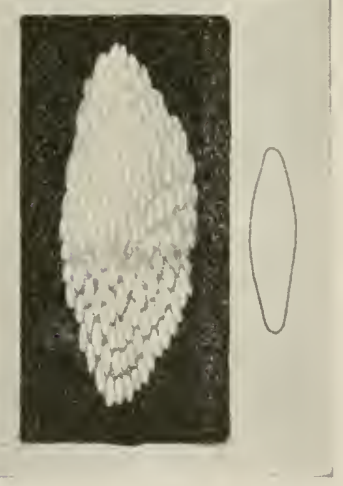

58 
Plate AV.

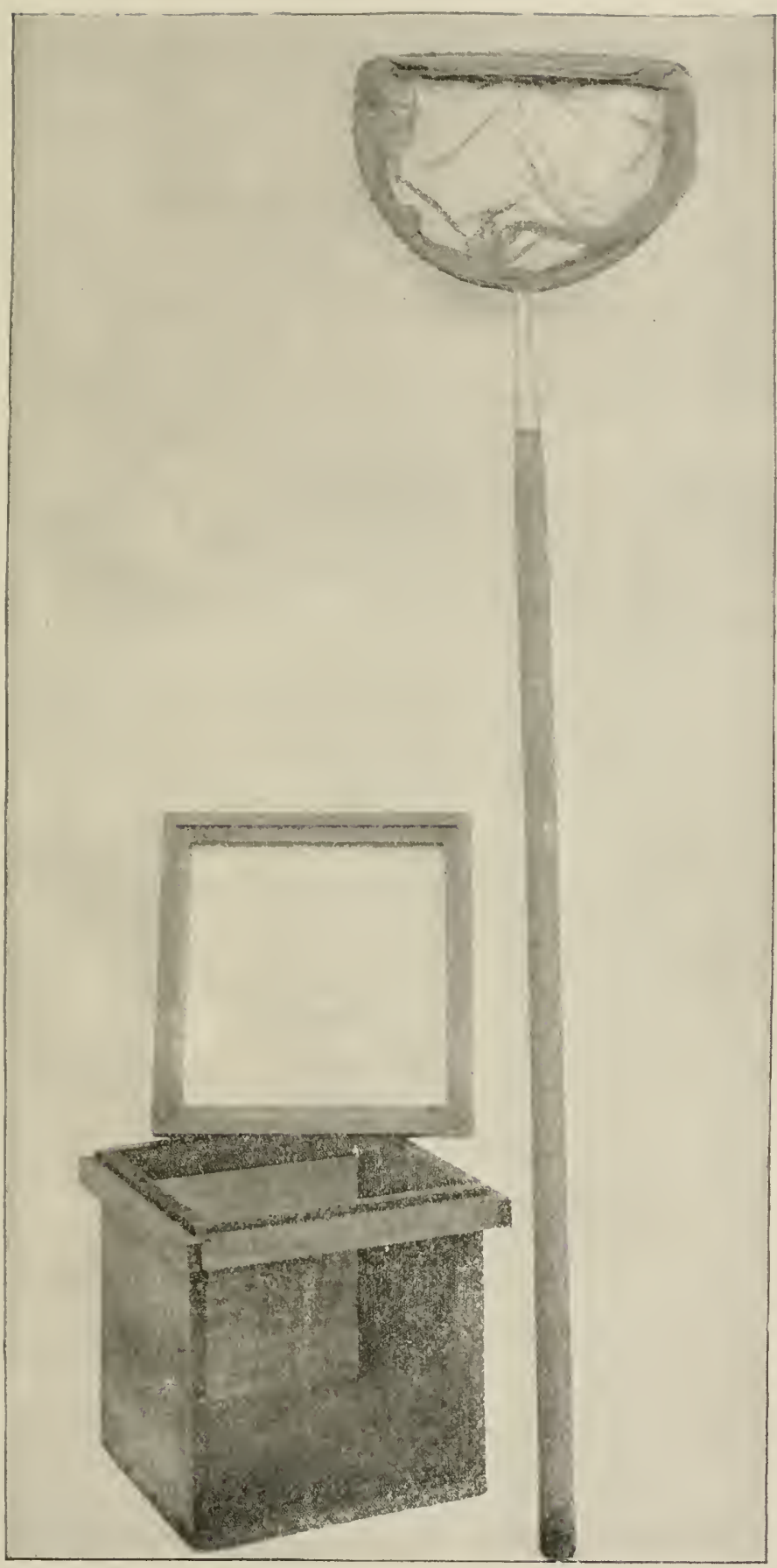


PIATE TYII.

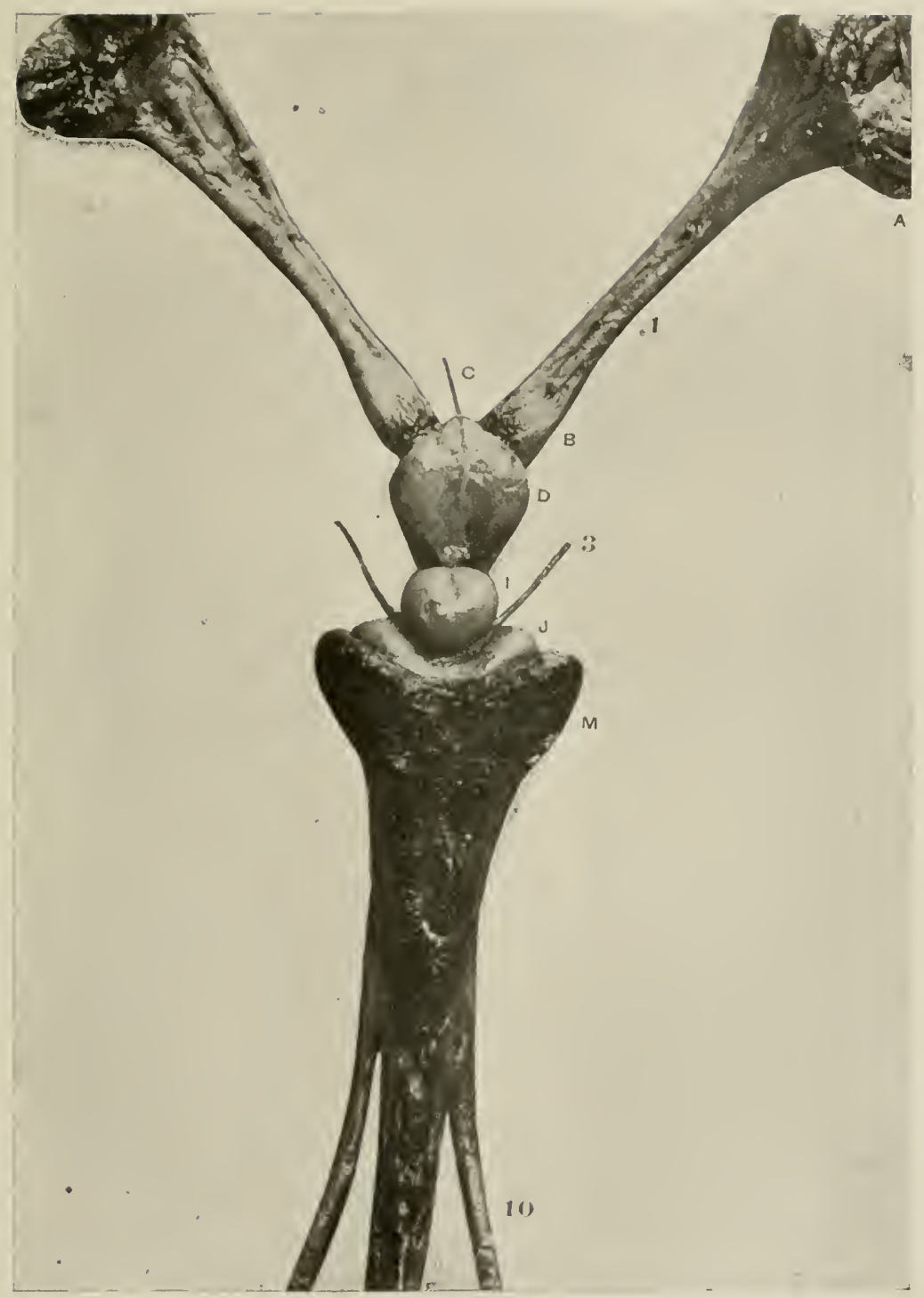

Fir. 2. 


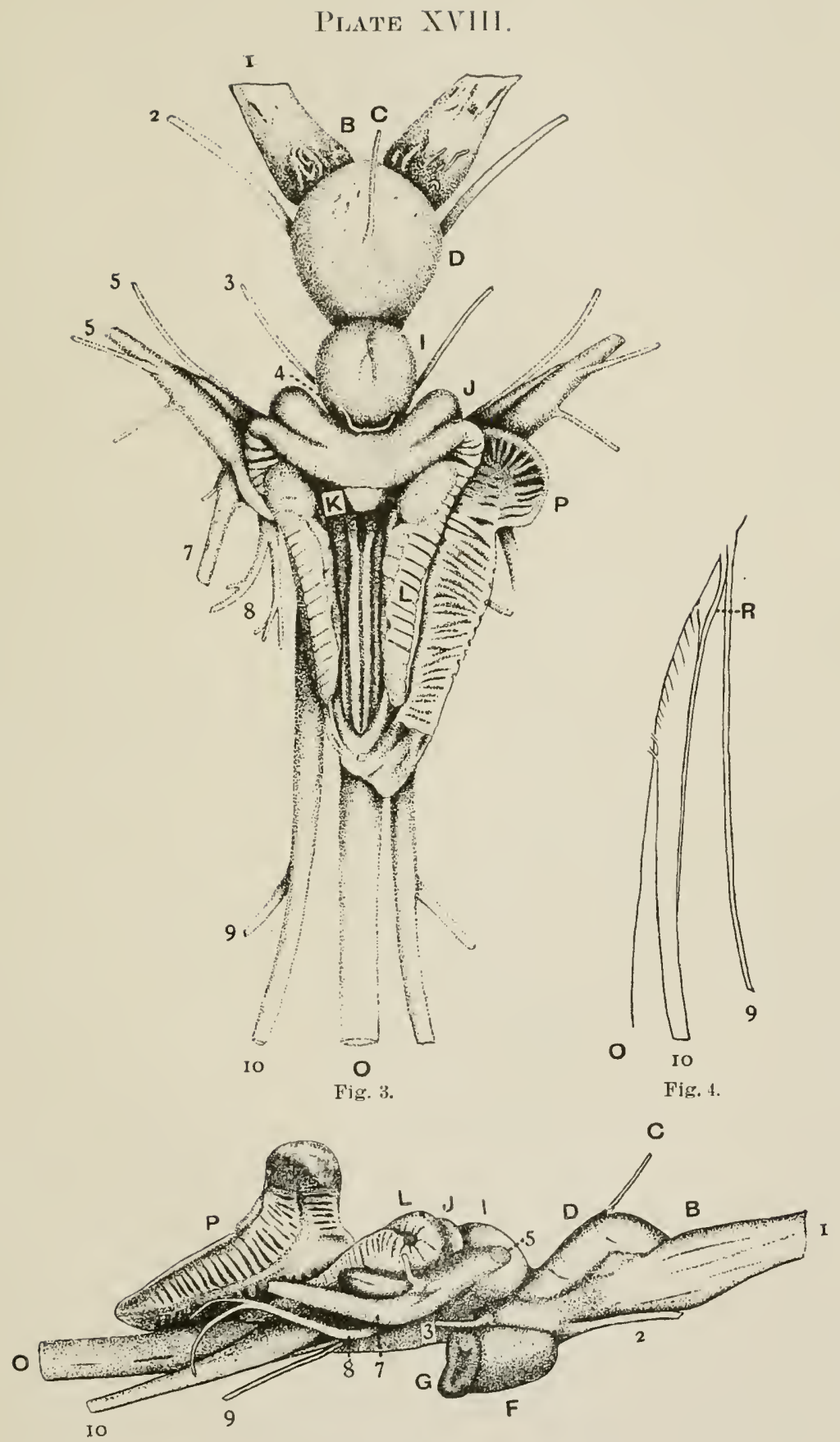

Fig. 5. 
PLATE NIX.

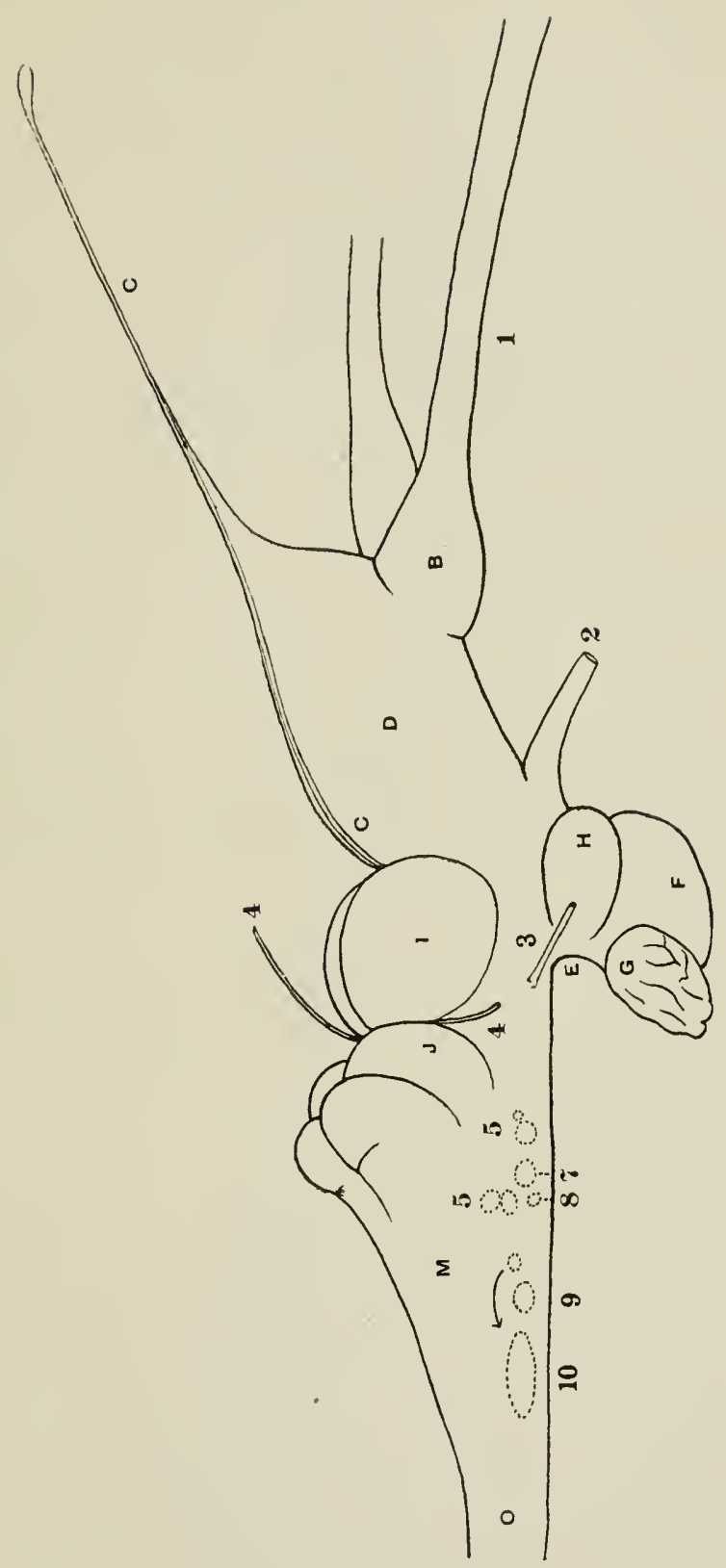

fig. 6. 


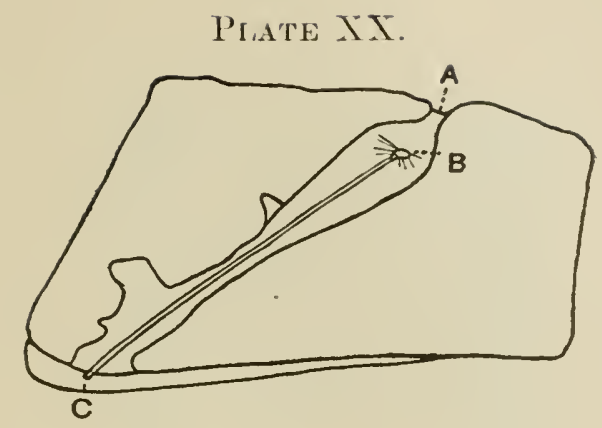

Fig. 7.

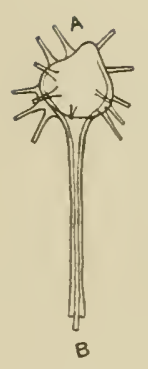

Fig. 8.
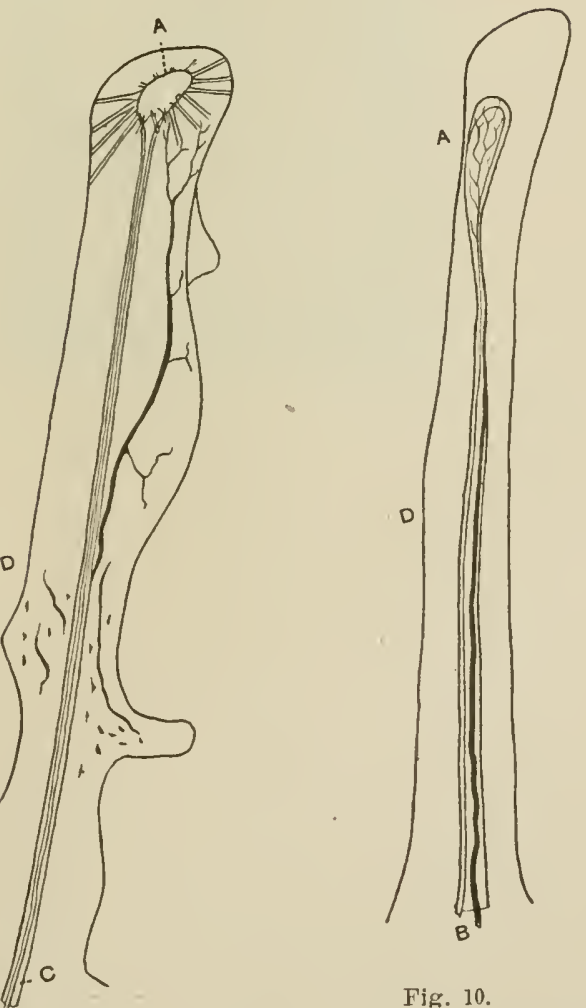

Fig. 10.

Fig. 9. 


\section{Plate NXI.}

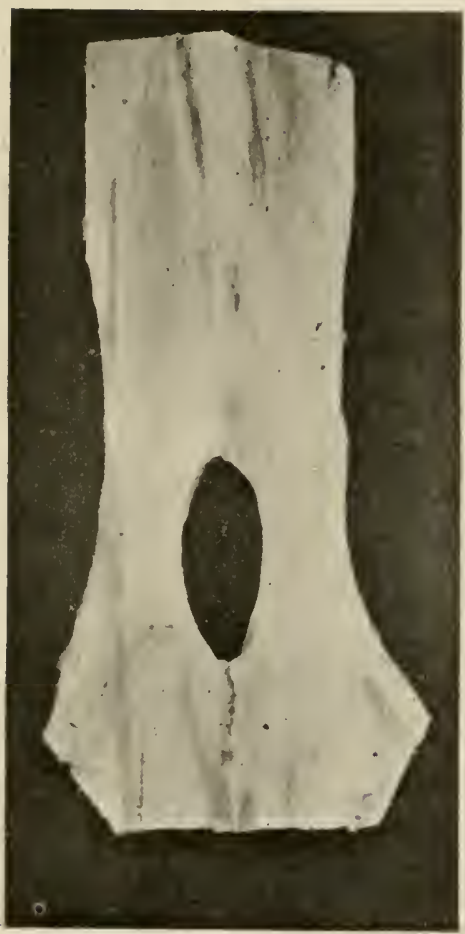

Fig. 11. 
PLATE XXII.

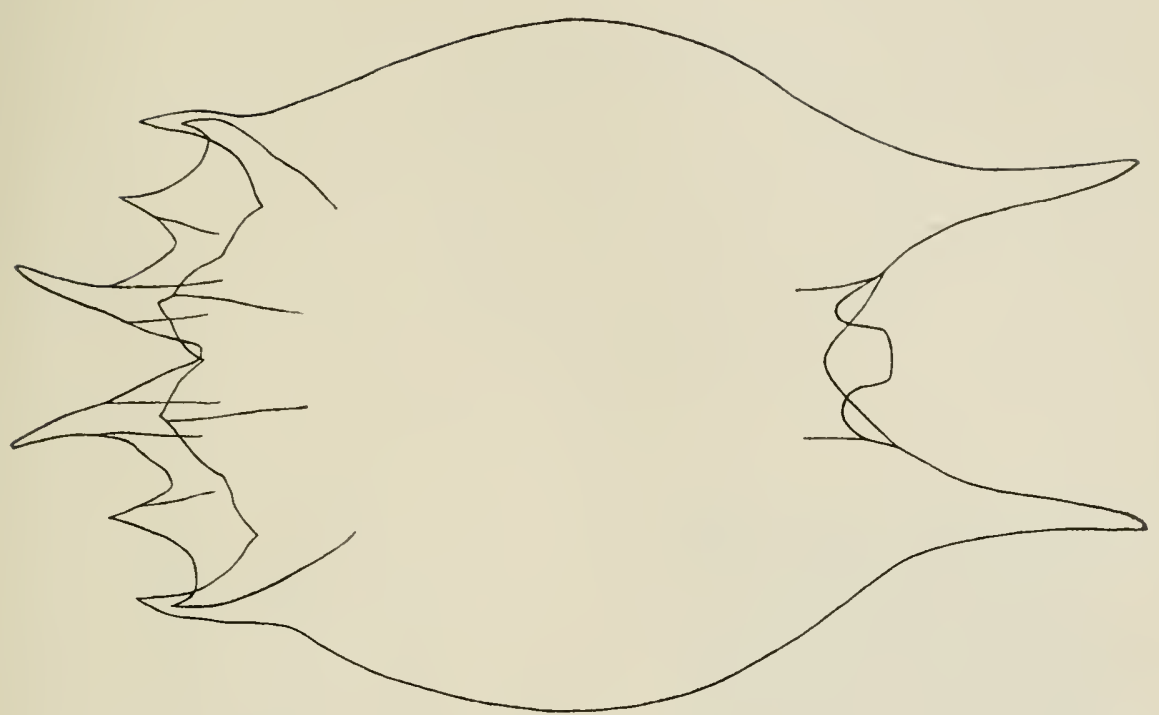

Fig. 1.

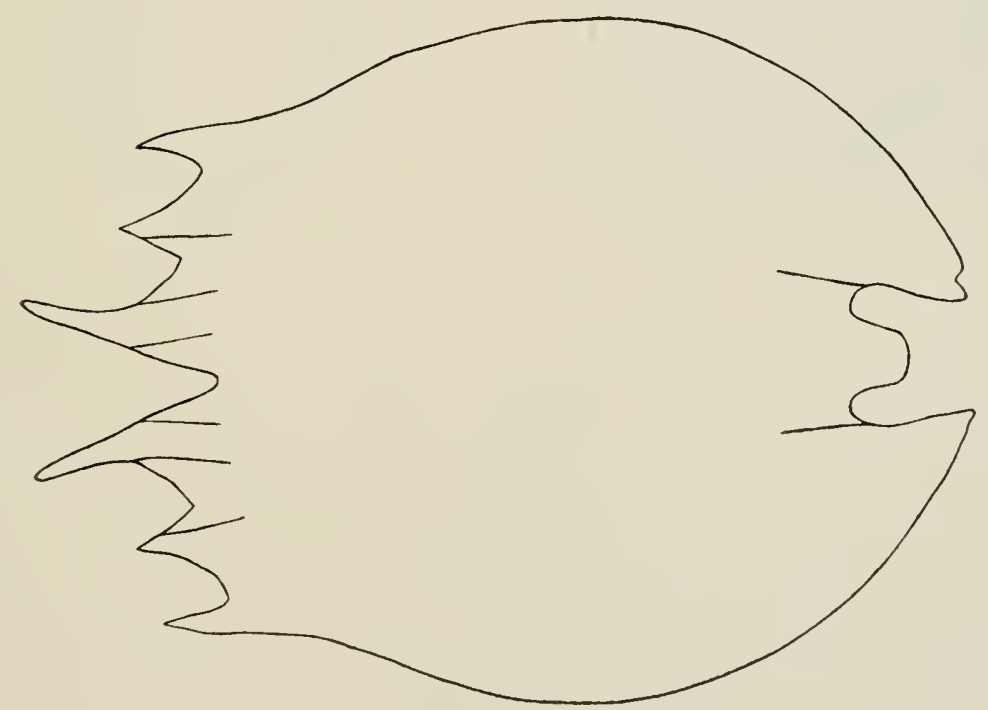

Fig. 2. 


\section{Plate IXIII.}

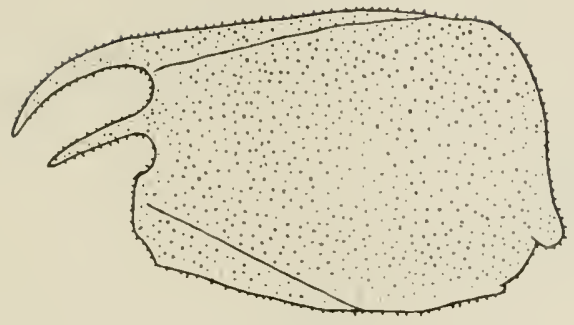

Fig. :3.

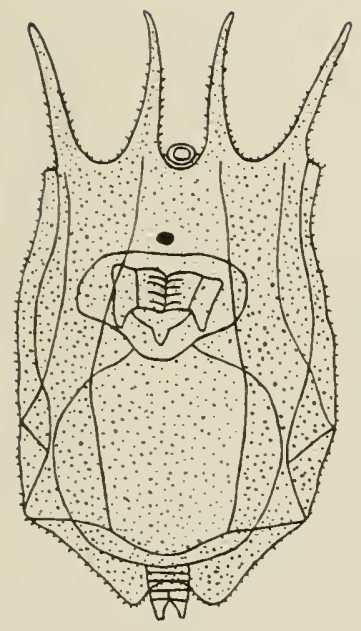

Fig. 4.

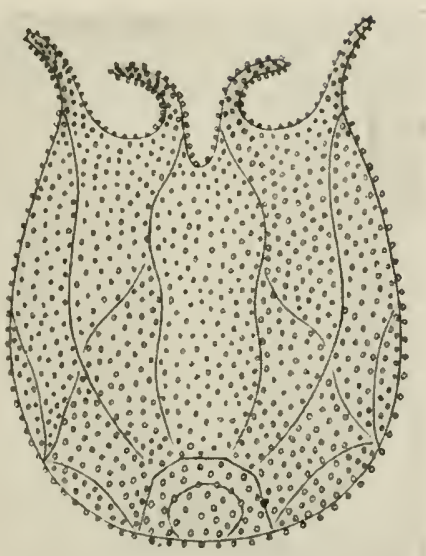

Fig. 6.

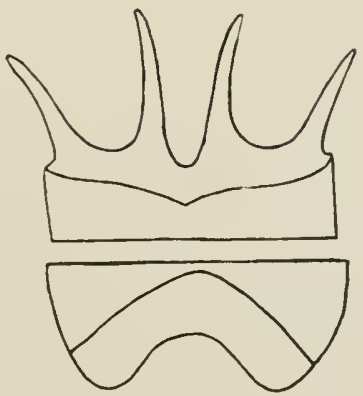

Fig. $\bar{~}$ 


\section{Plate XXiv.}

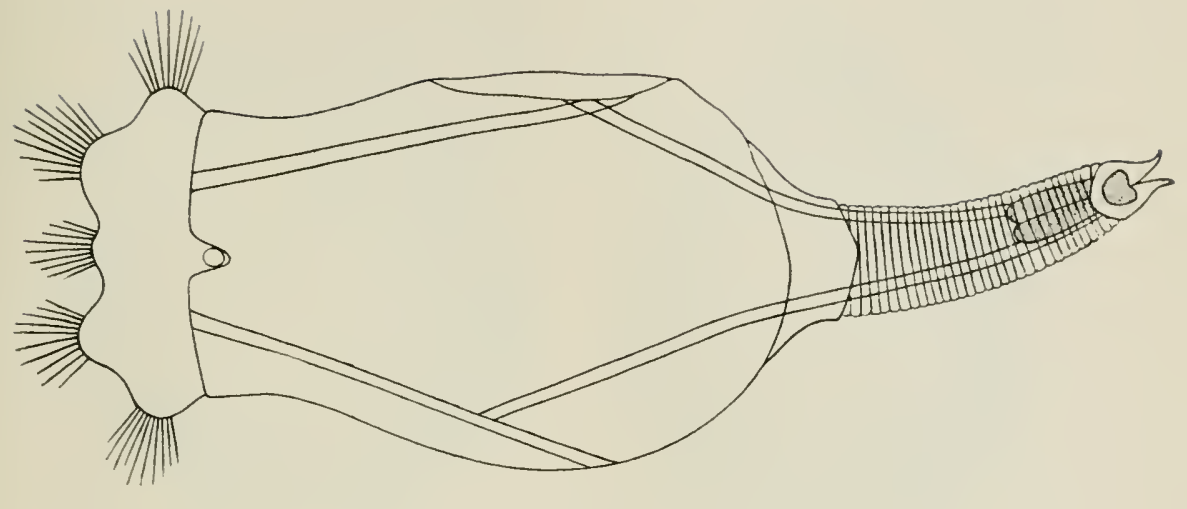

Fig. 7.

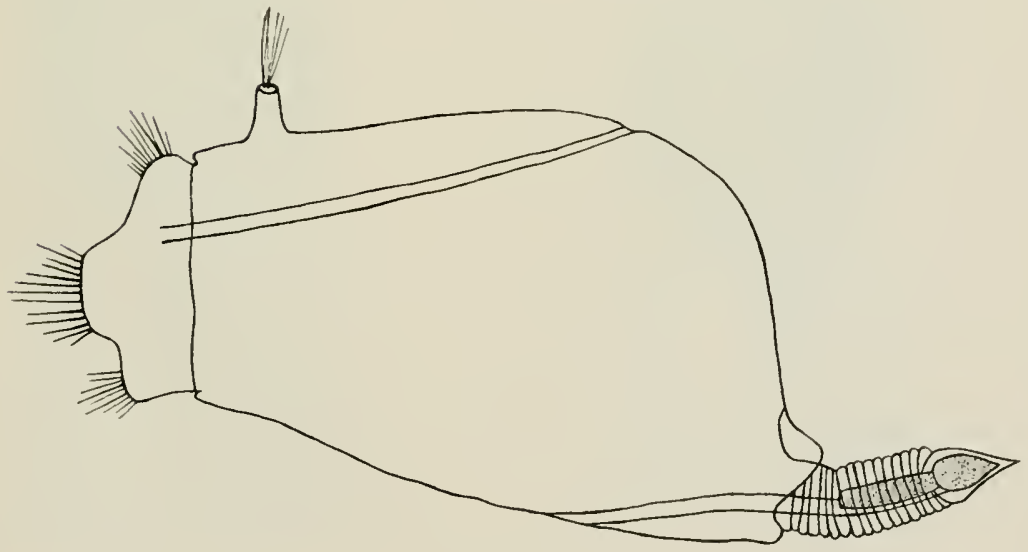

Fig. 8. 
Plate NXY.

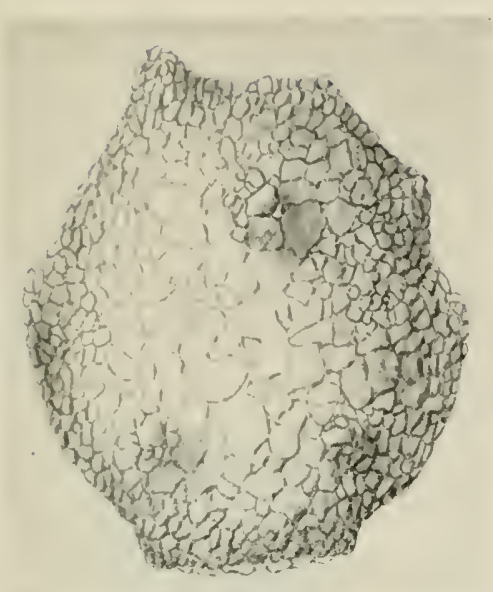

Fig. 9.

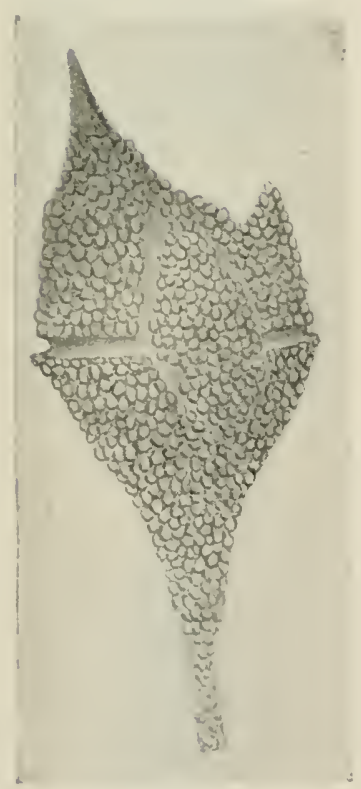

Fig. 11.

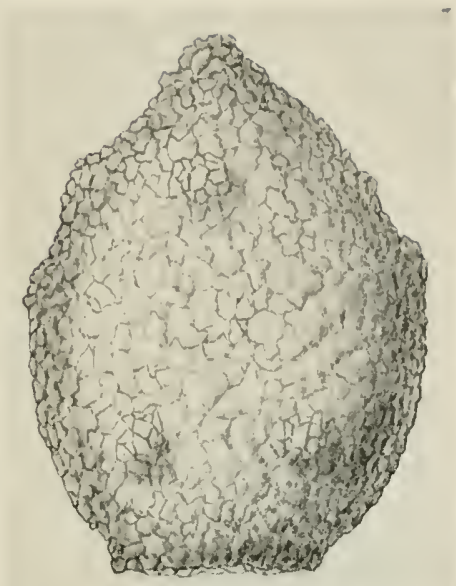

Fig. 10.

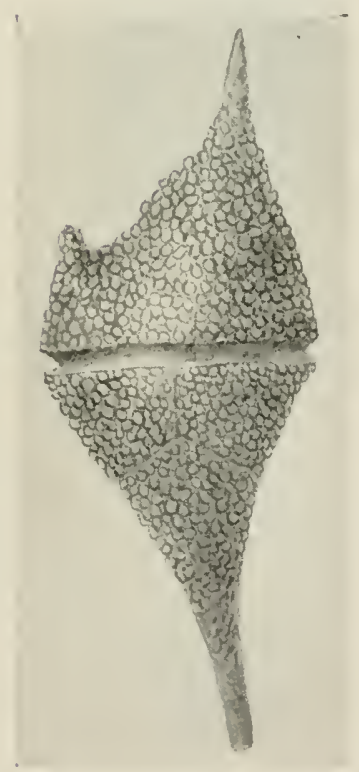

Fig. 12. 


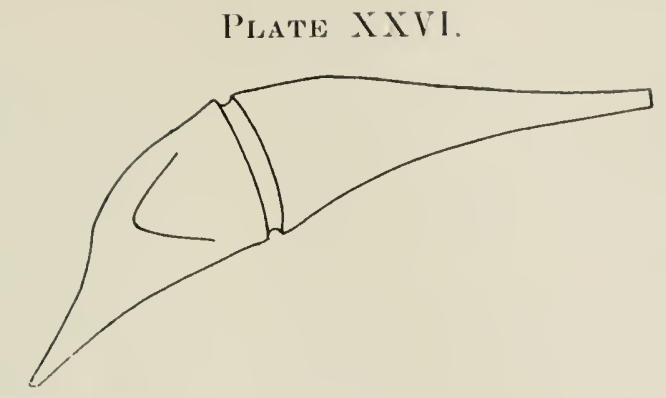

Fig. 13.

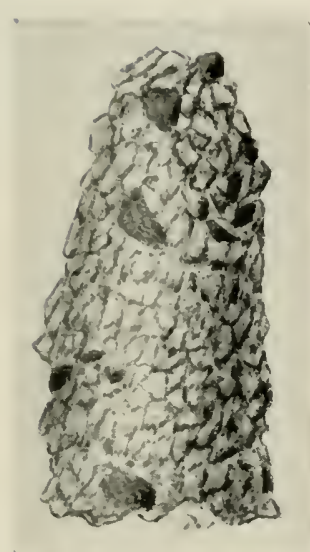

Fig. 14 .
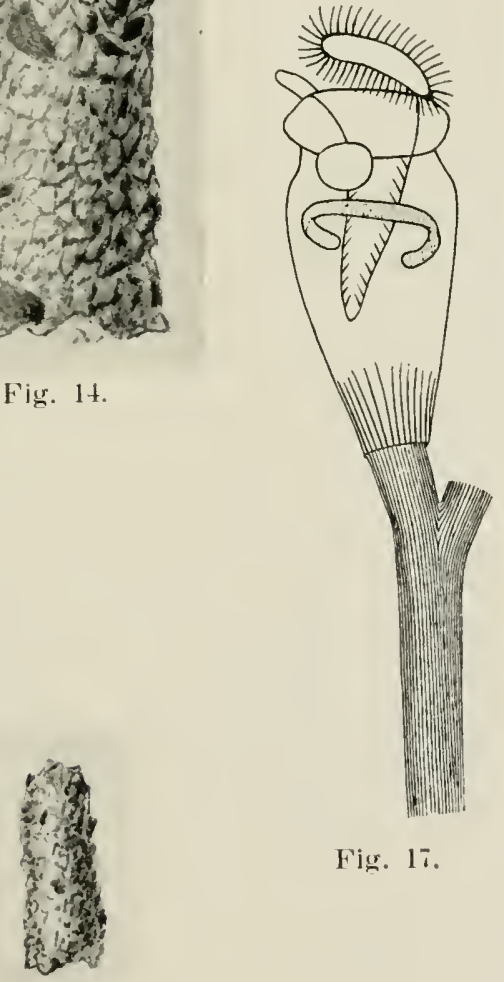

Fig. 17.

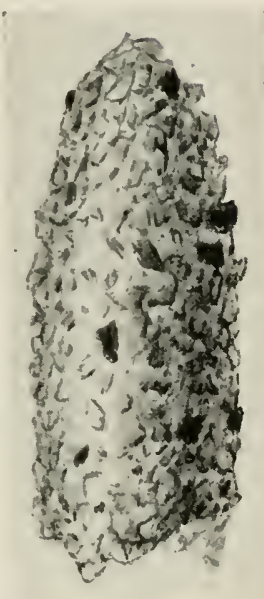

Fig. 15.

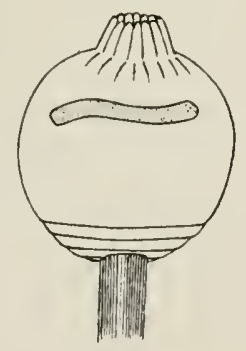

Fig. 18.

Fig. 16 . 


\section{PLATE IXVII.}

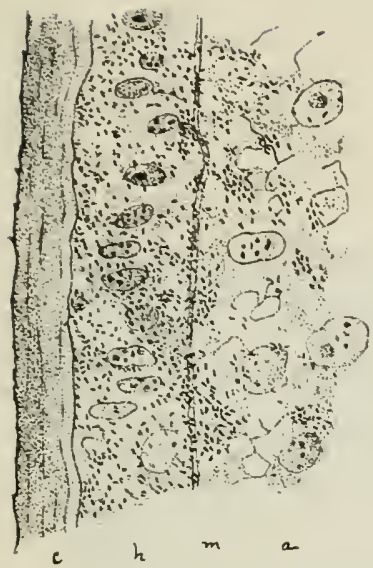

Fie. 2.

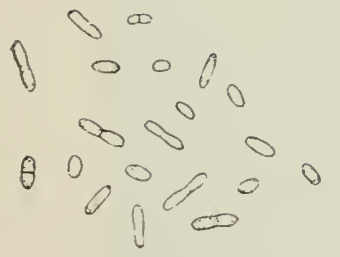

Fig. 1

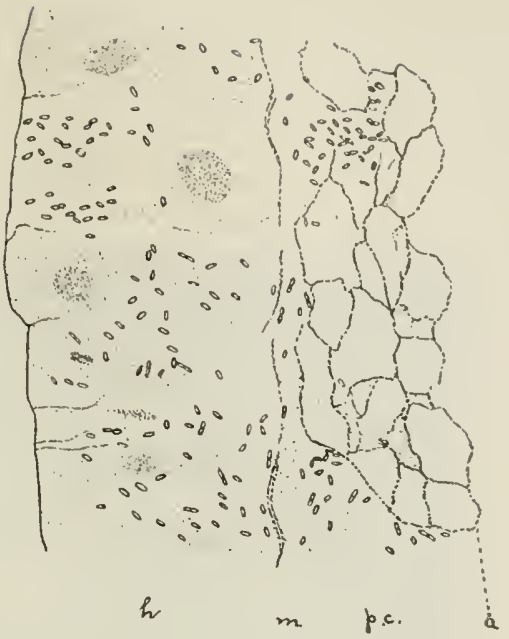

Fig. 3 .

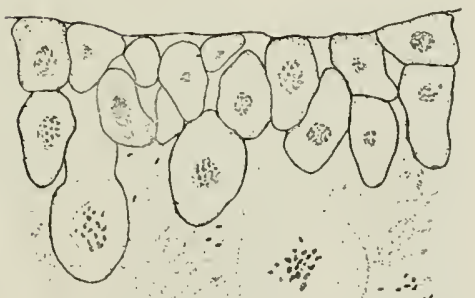

$\therefore \div$

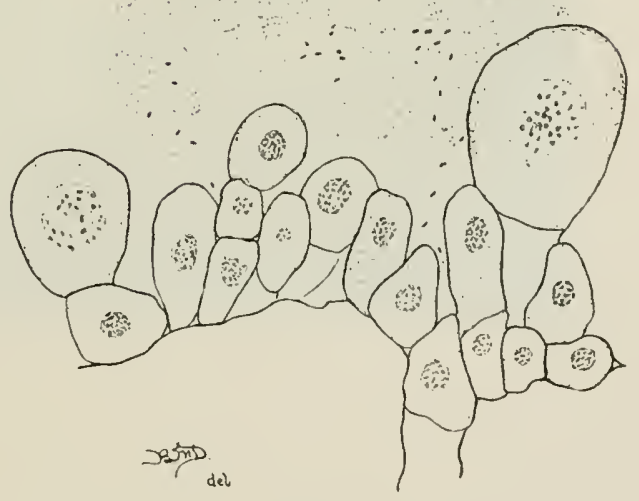

Fig. 4. 
Plate XXVih.

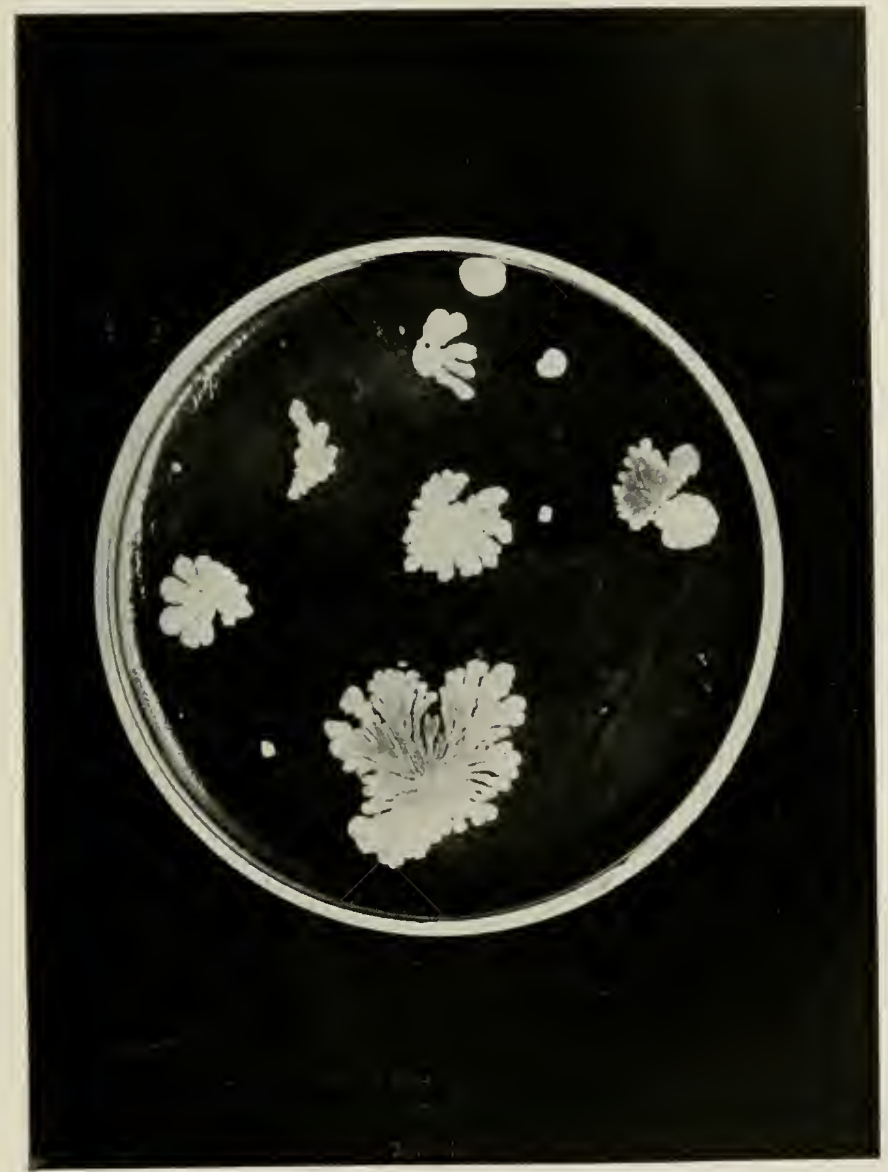

Fiv. 5. 


$$
\%
$$


Plate XIX.

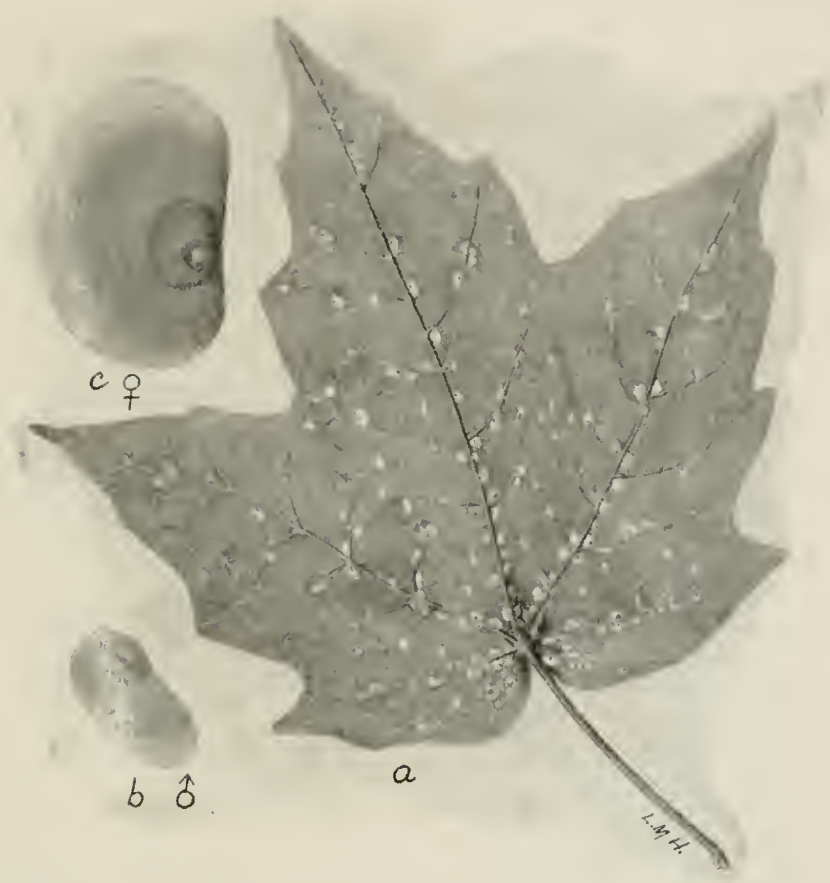

Fie. 1.

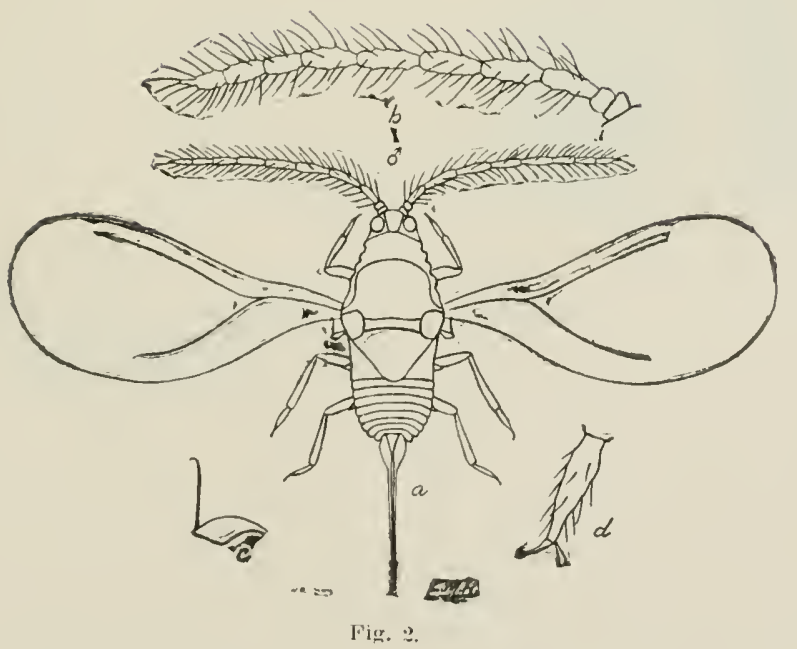


Plate XXXi.

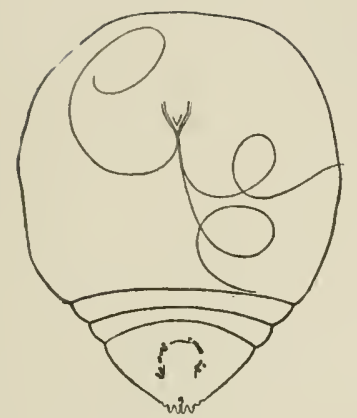

Fig. 1.

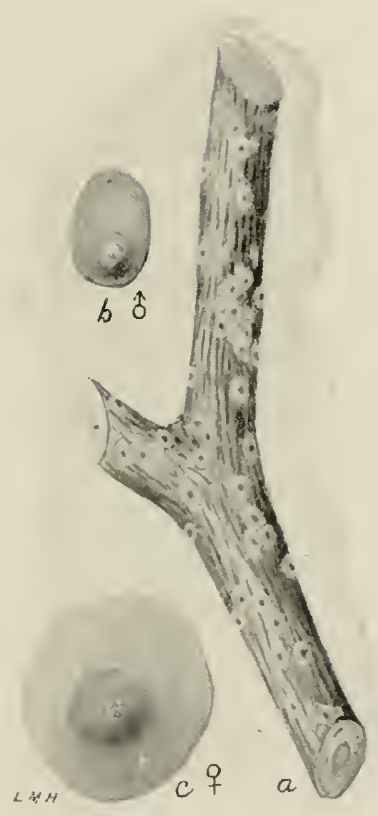



ஓं क्ष

कृ

Fig. 3.

Pिद्ध

Fig. :. 


\section{Plate XXYil}

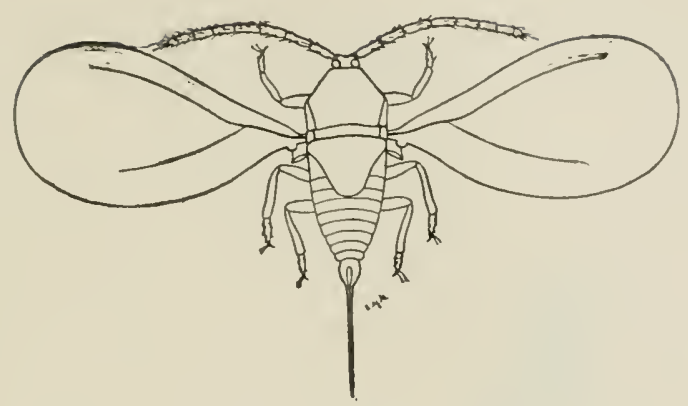

Fig. 1.
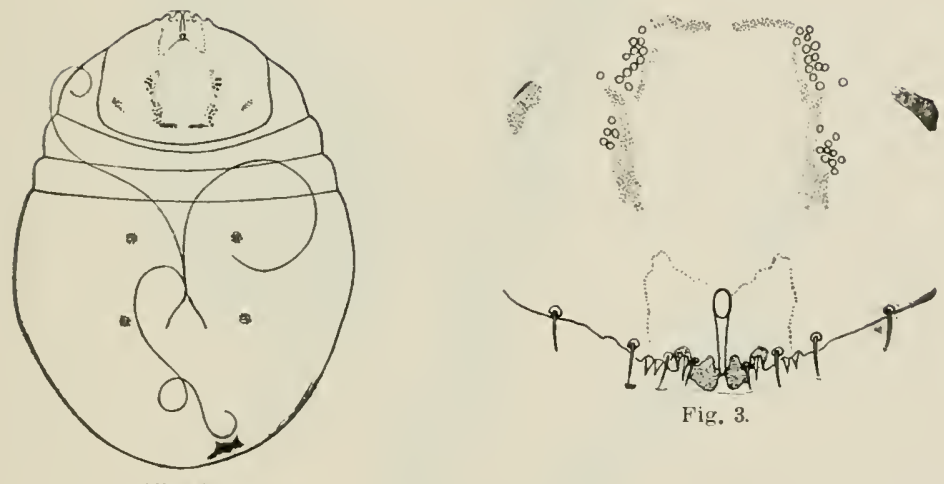

Fig. :.
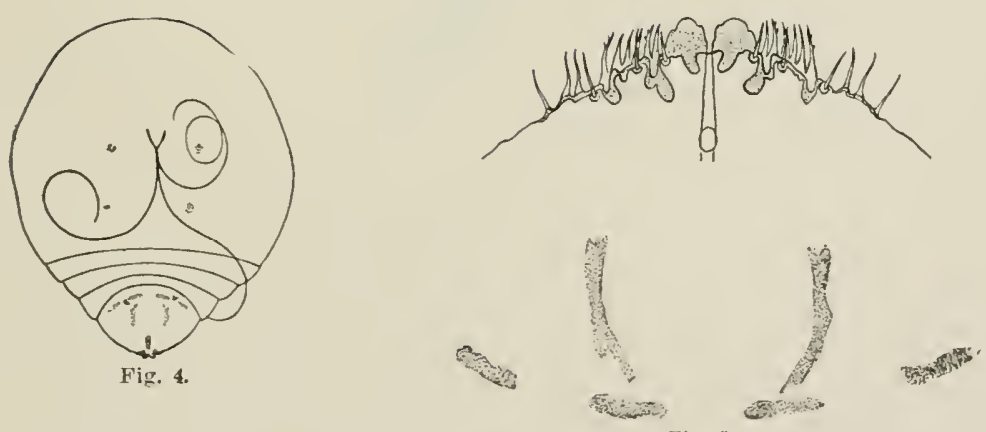

Fir. 5. 


$$
\text { of }
$$




\section{Plate IXIIV.}

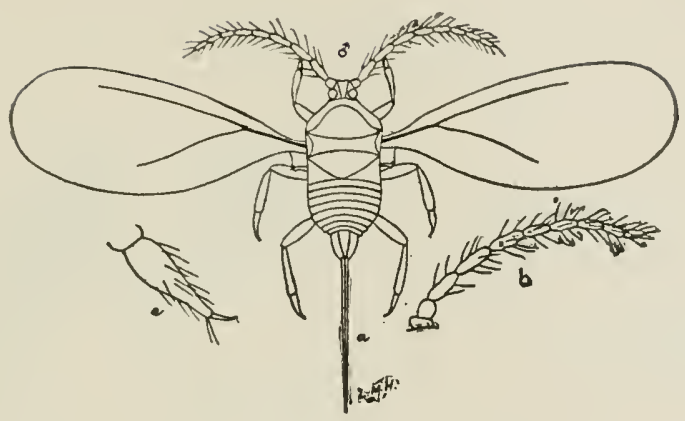

fier. 1.

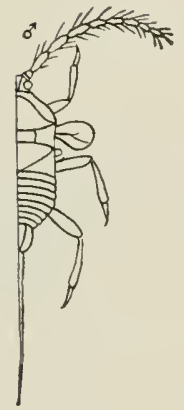

Fig. 2.

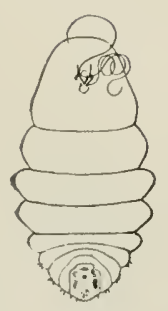

Fig. 4.

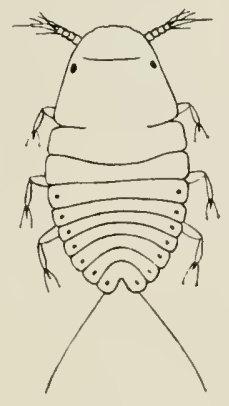

Fig. 3.
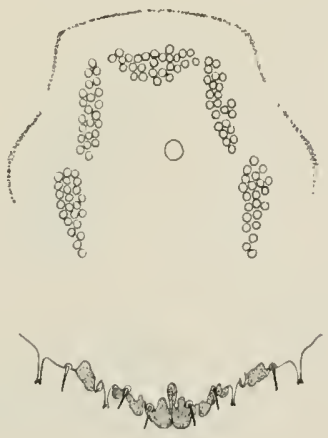

Fig. 5. 


\section{P'ATE XXYY.}

g.s.gl.

1
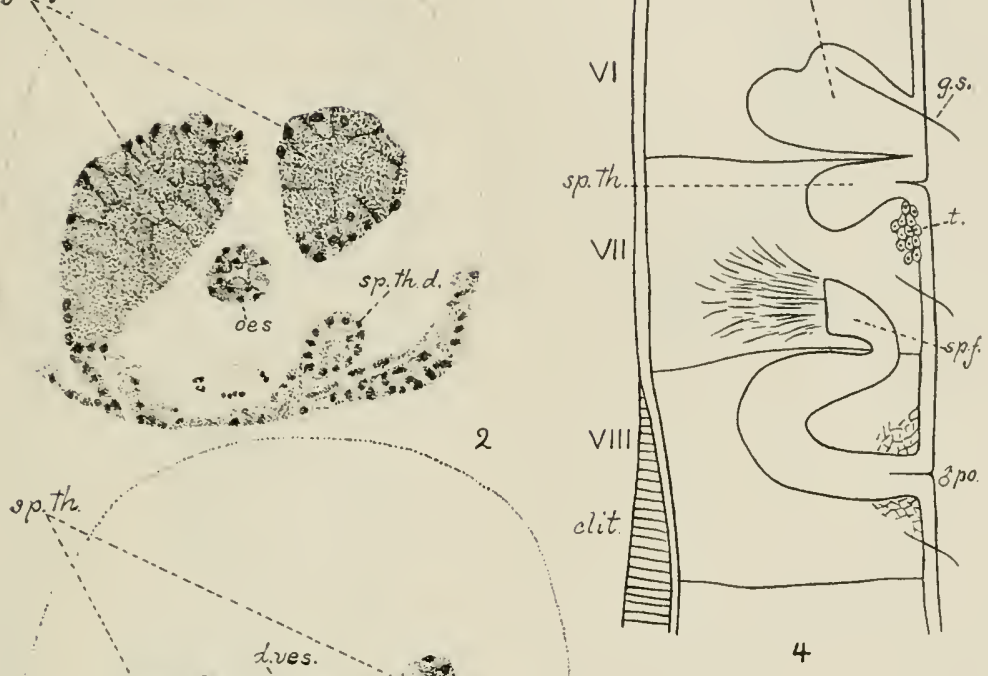

3

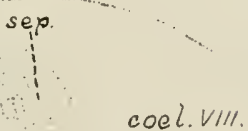

$\cos 2 . V / \%$
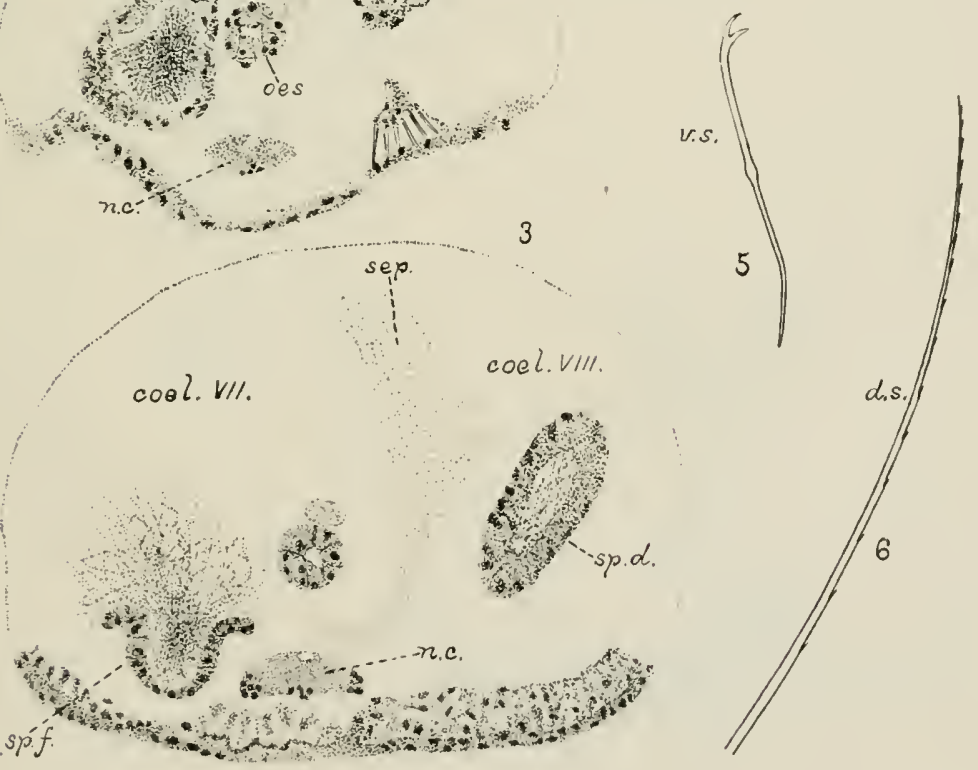
PLATE XIXVI.

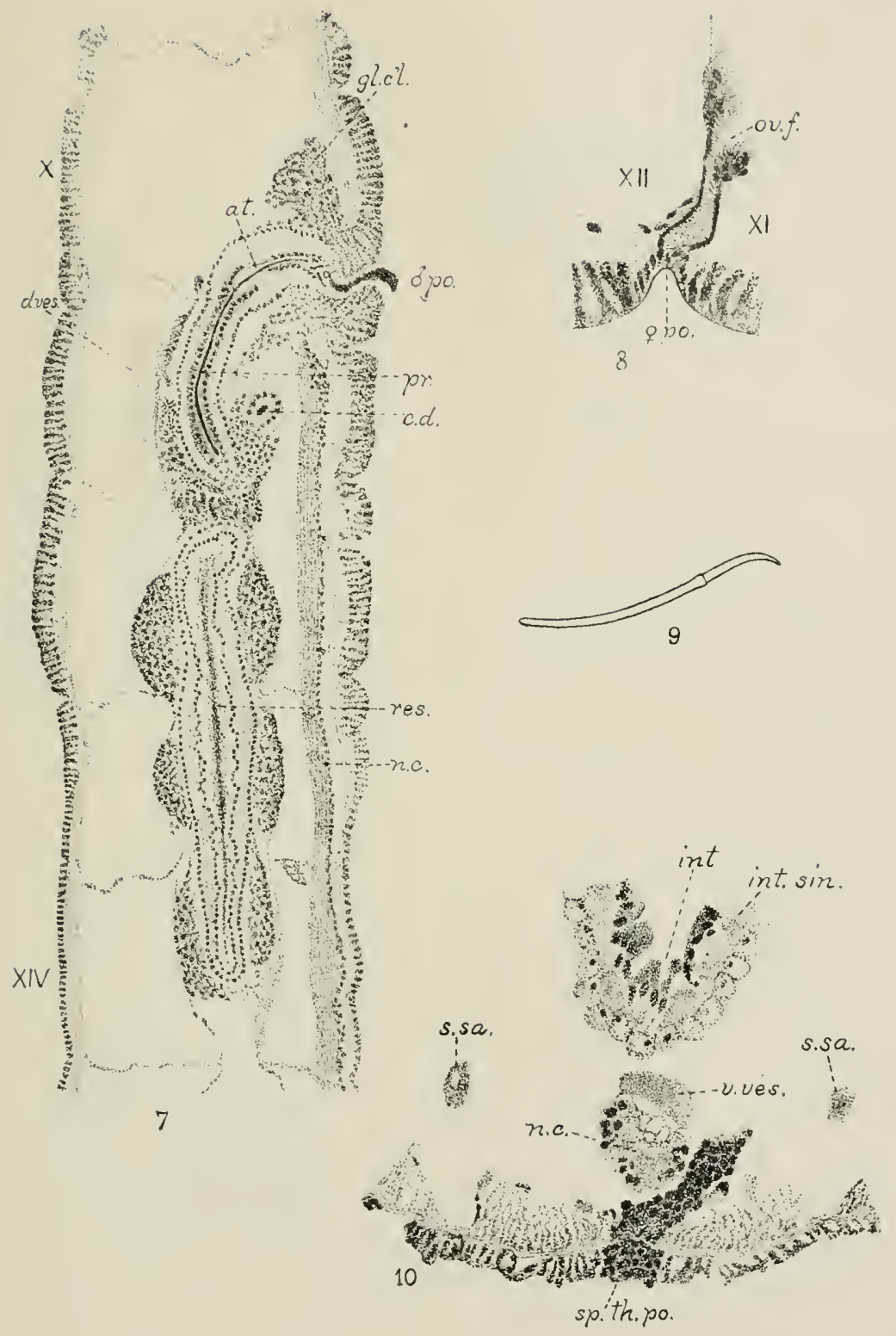




\section{Plate Xitill}
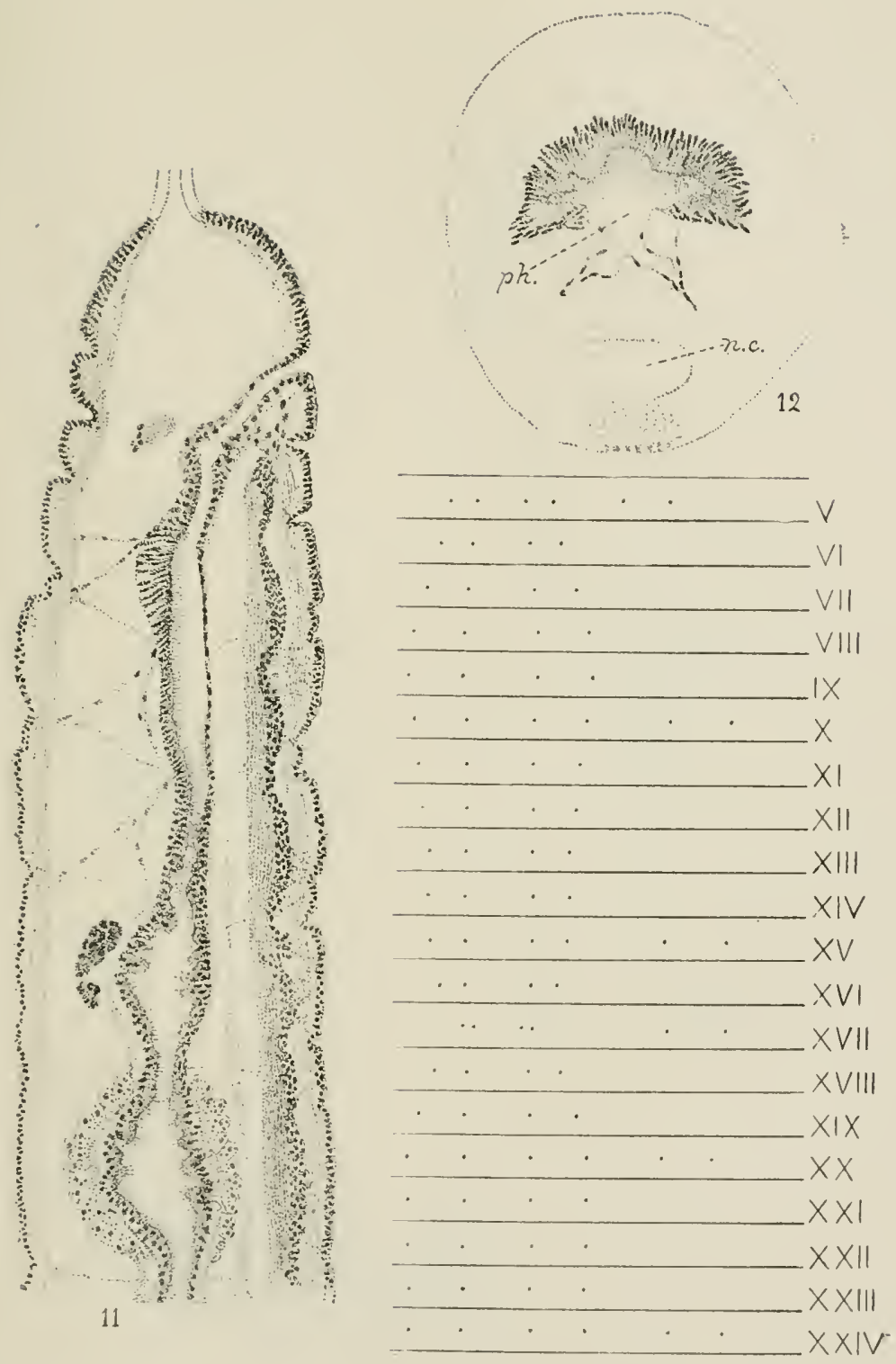


\section{Plate IXIVIII.}
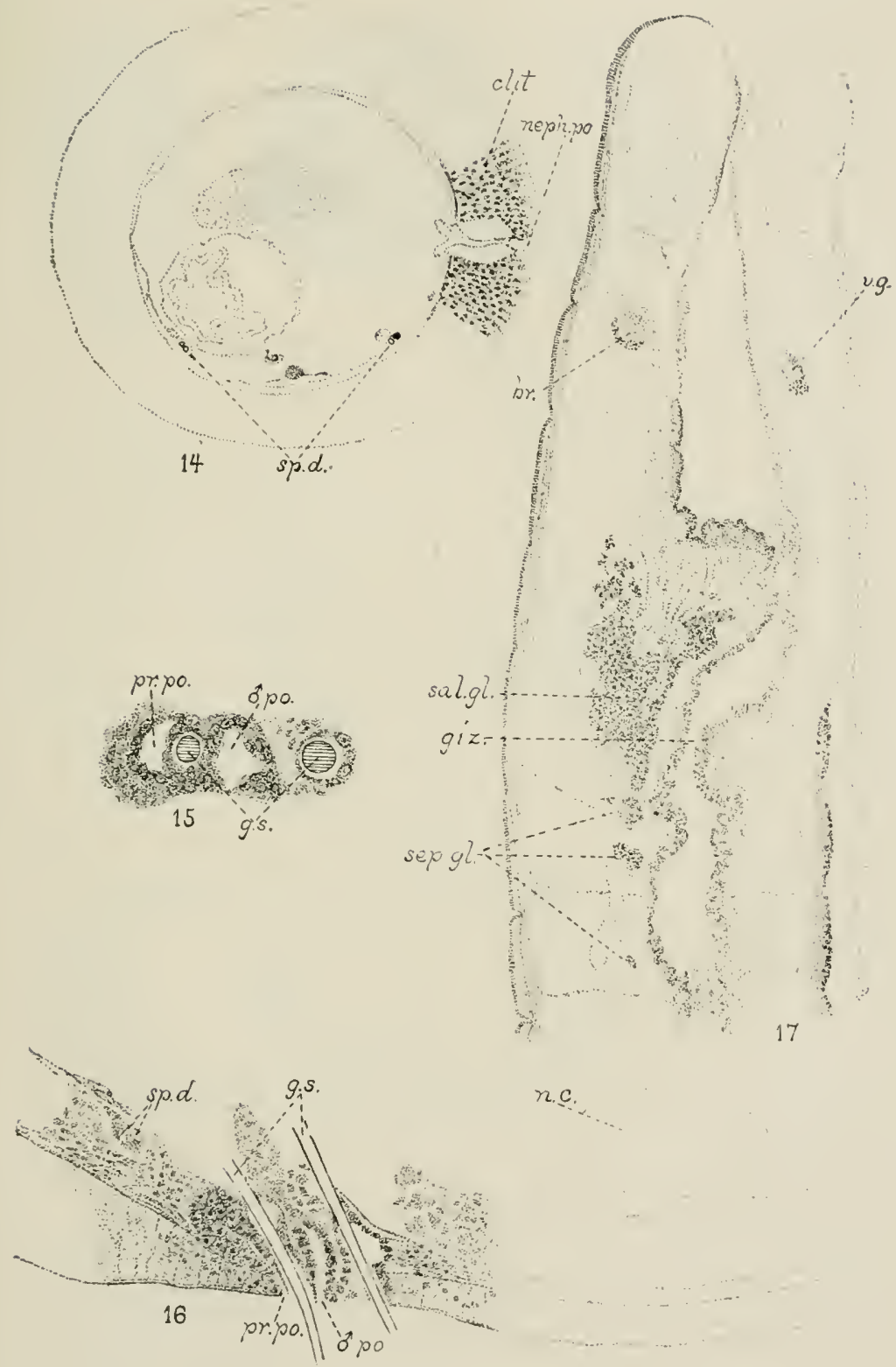

n.c. 
PLATE TXYIX.
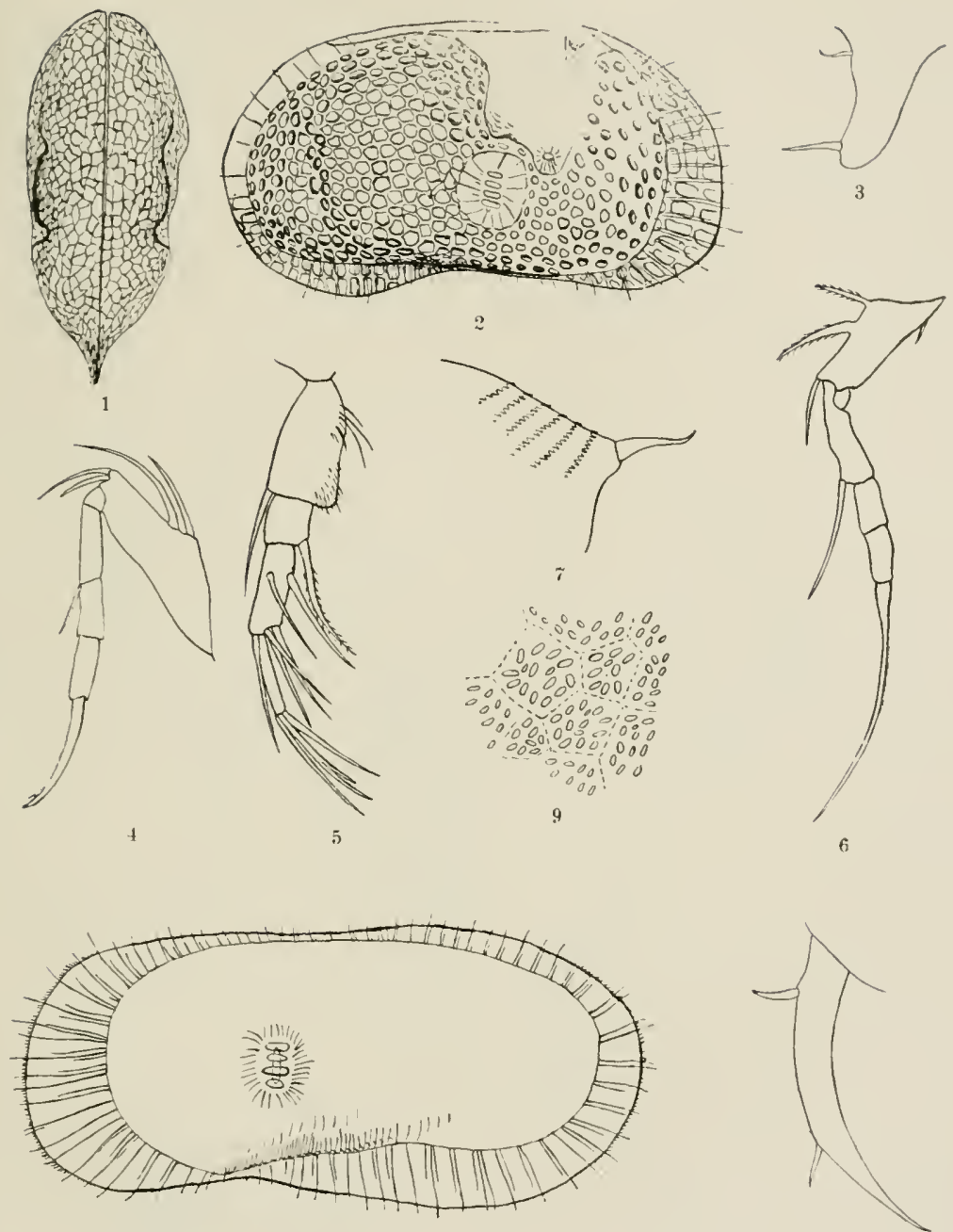

8

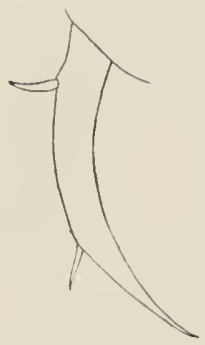

12
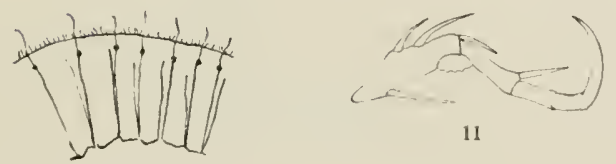

11

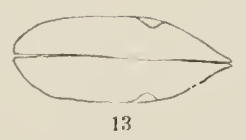




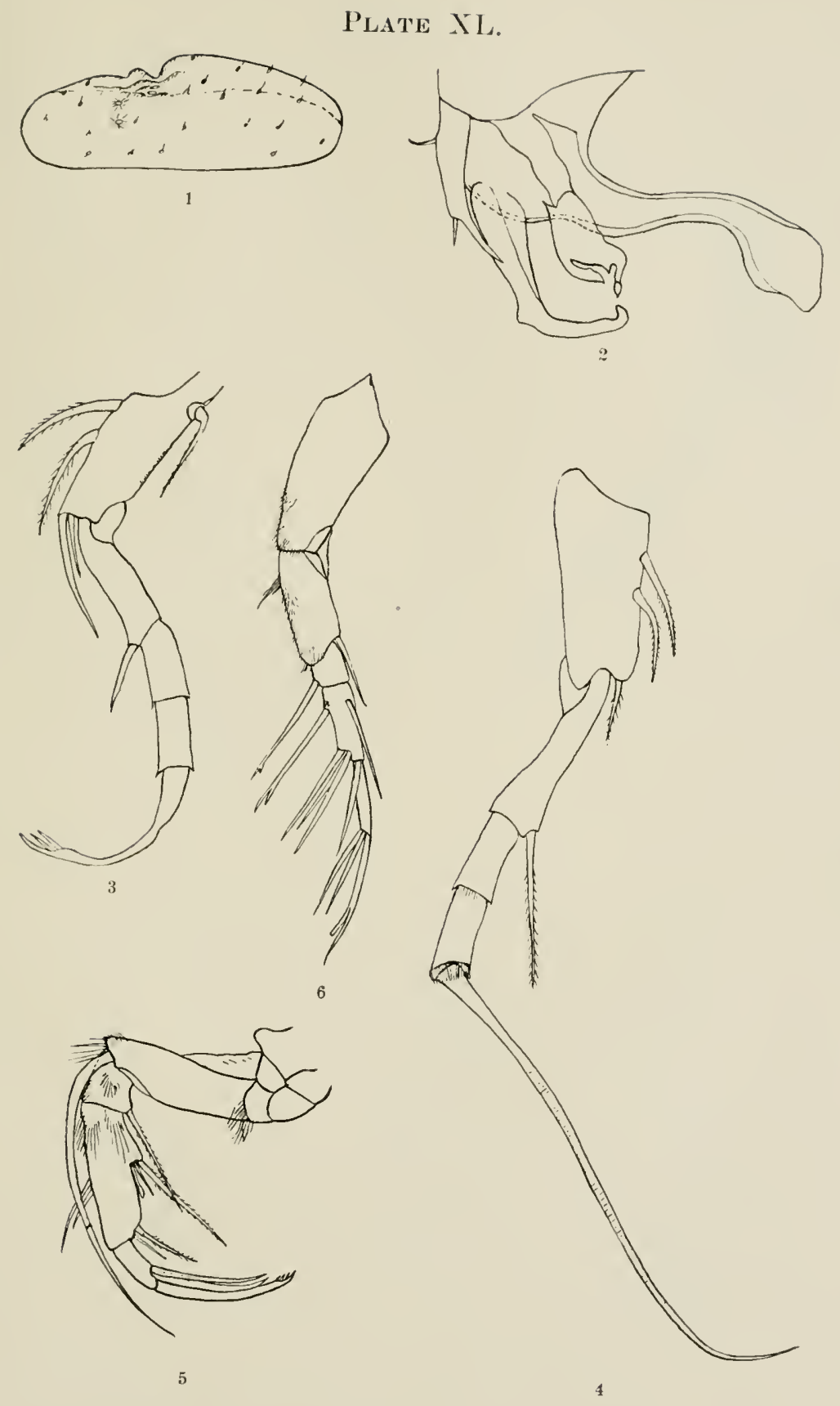


Plate Xli.
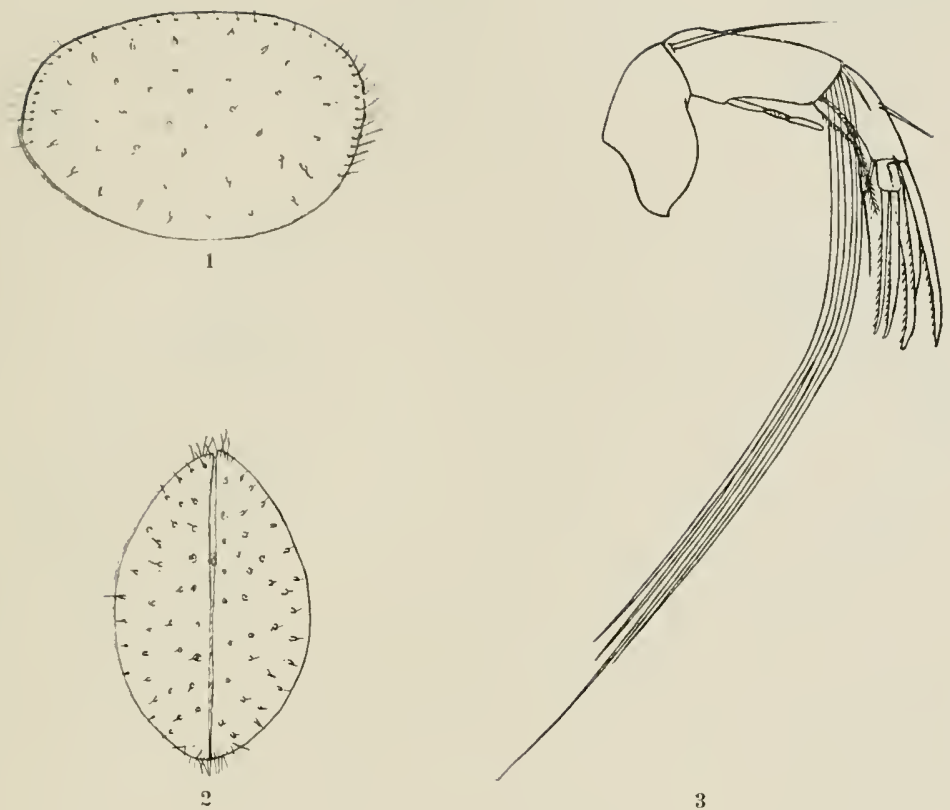

3
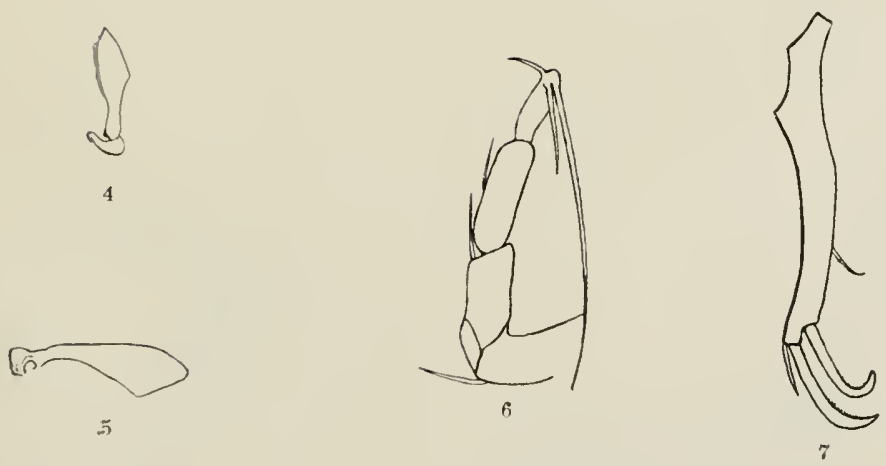
Plate XLII.
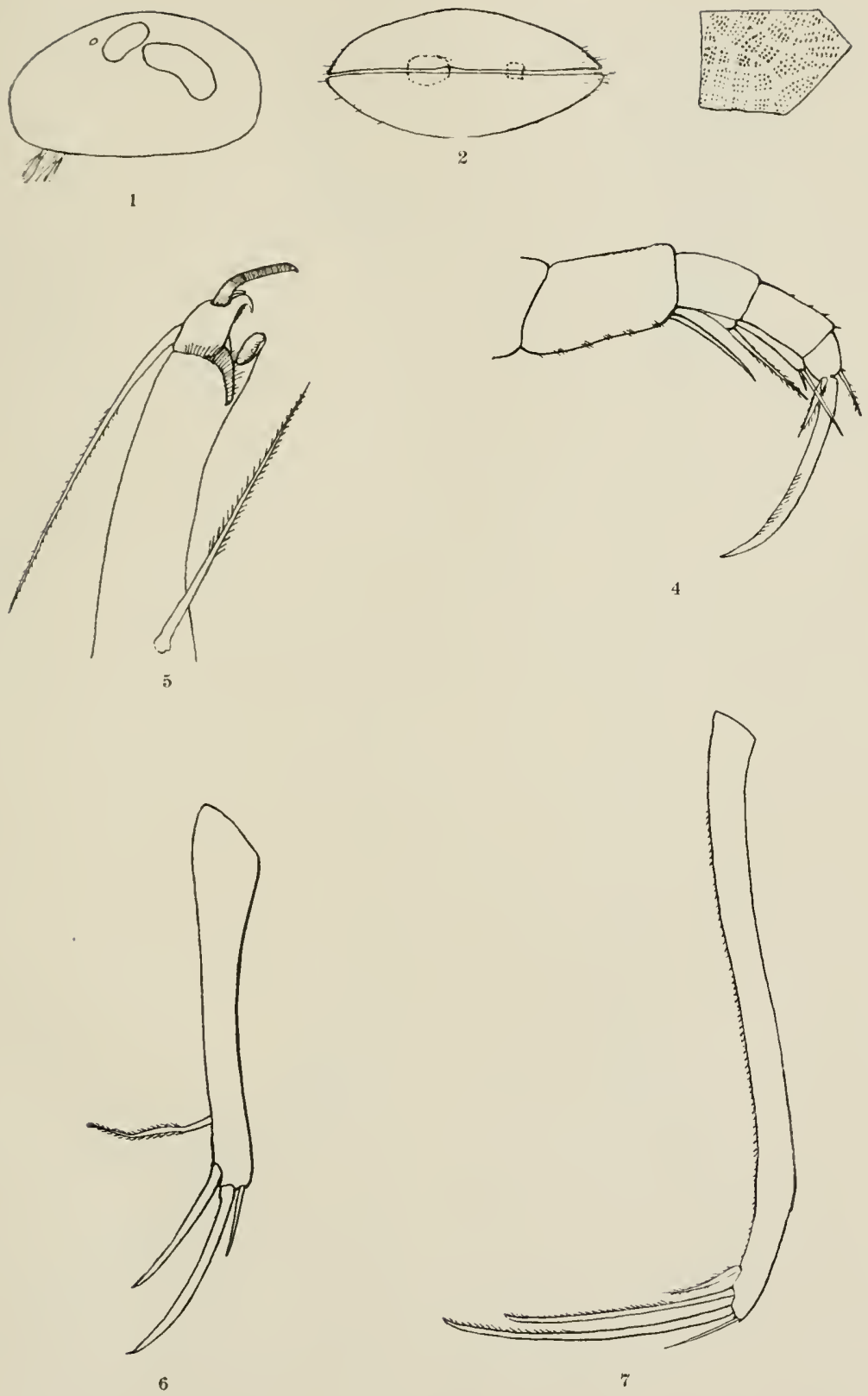
Prate xlili.

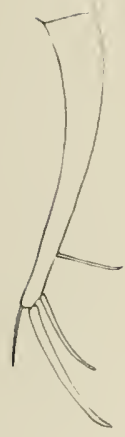

1

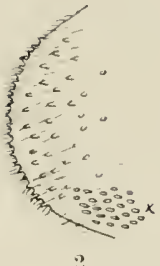

2

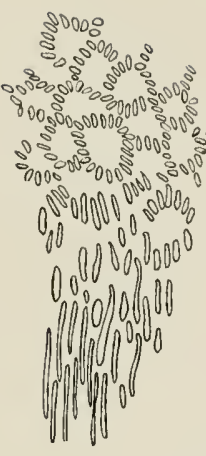

3

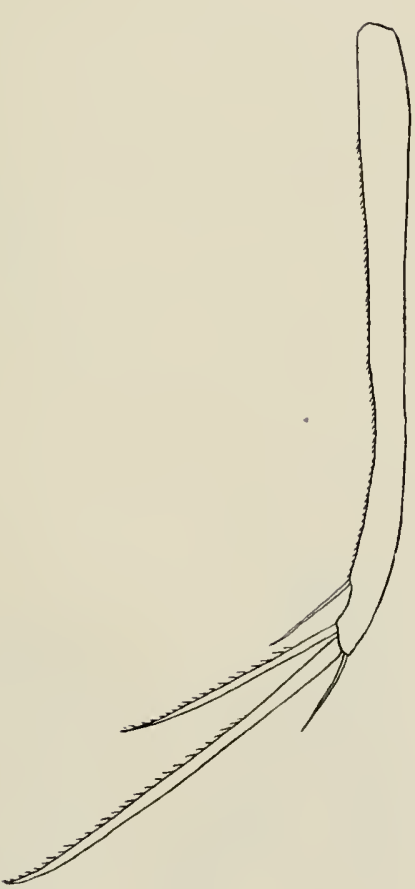

4

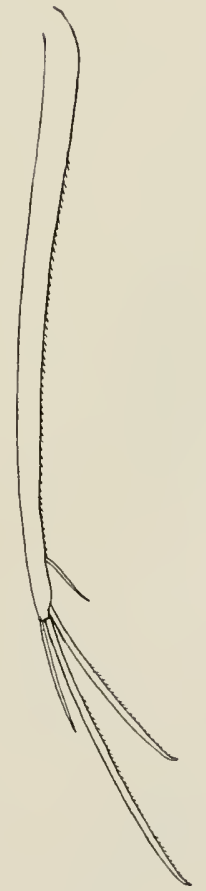

5 
Plate XliV.
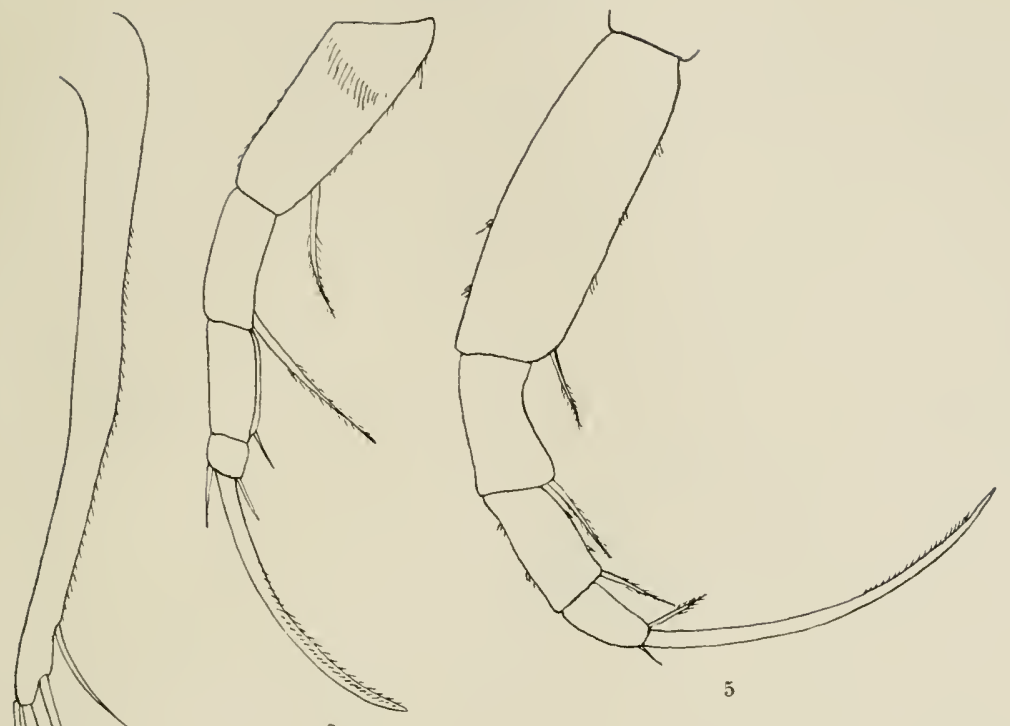

2

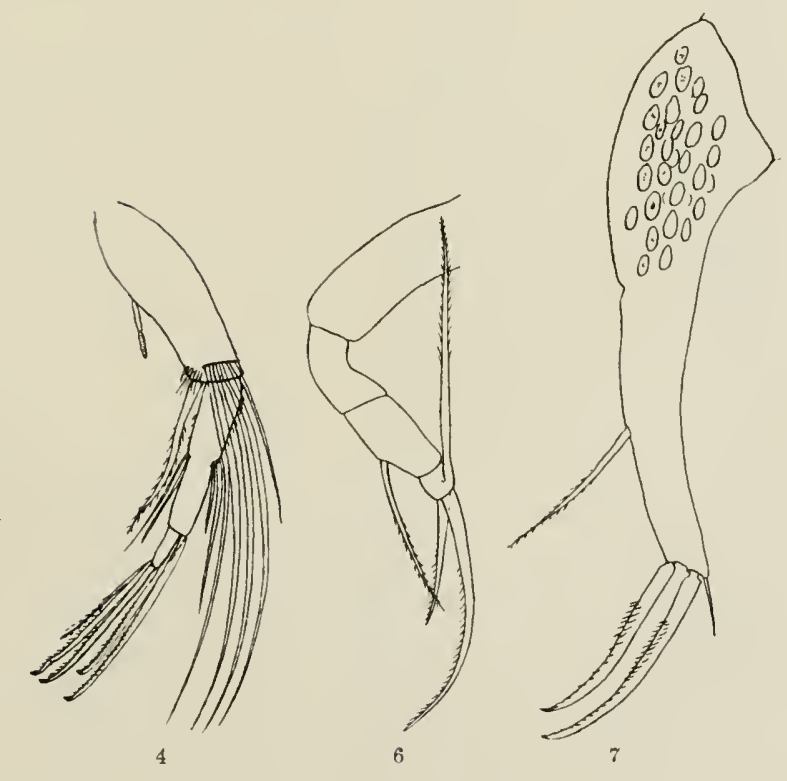


Plate Nis.
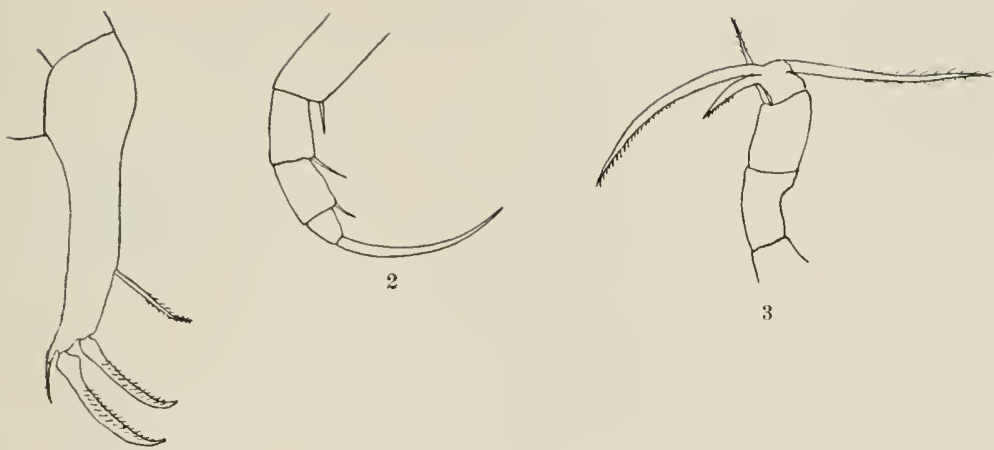

I
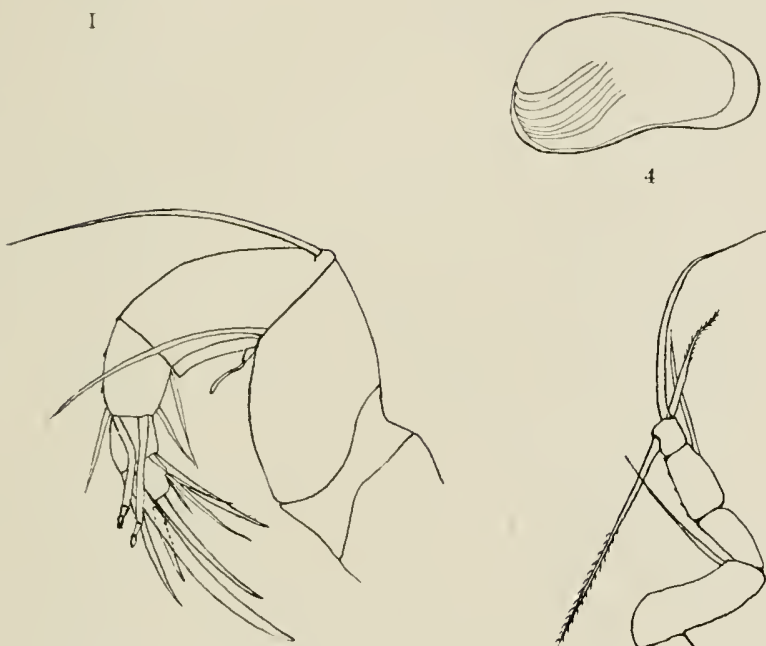

5
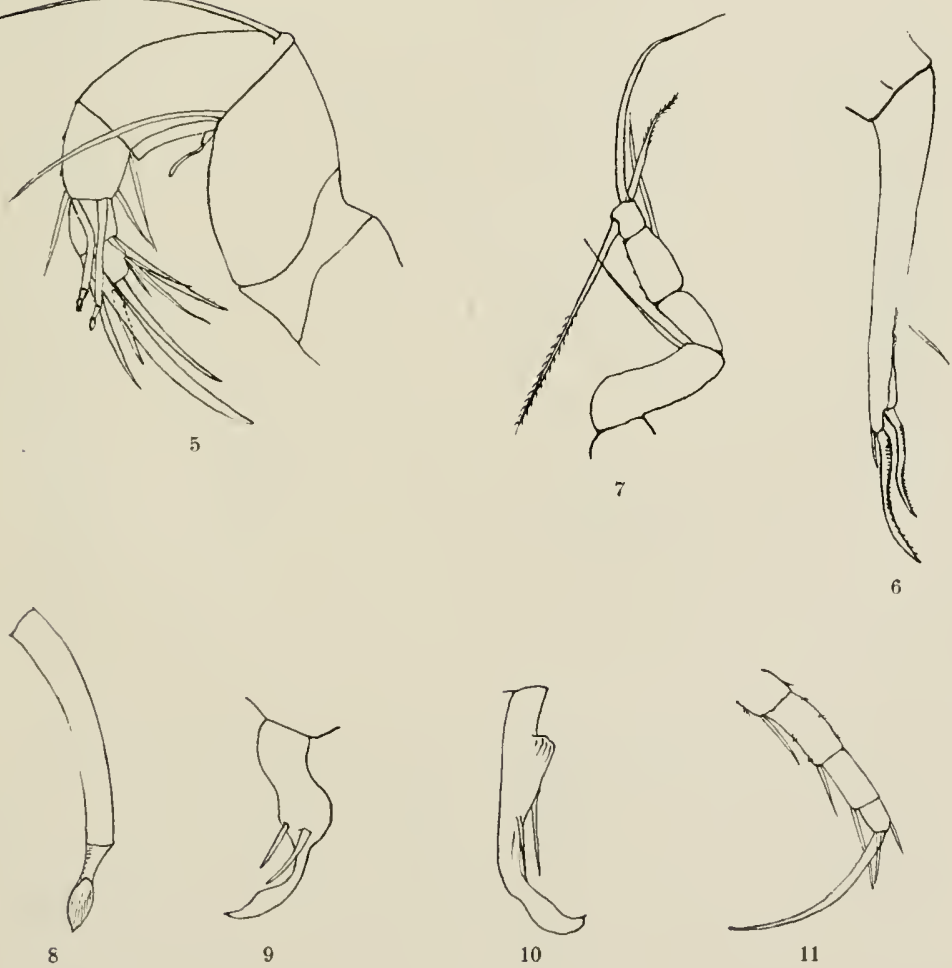
P'ATE XLII
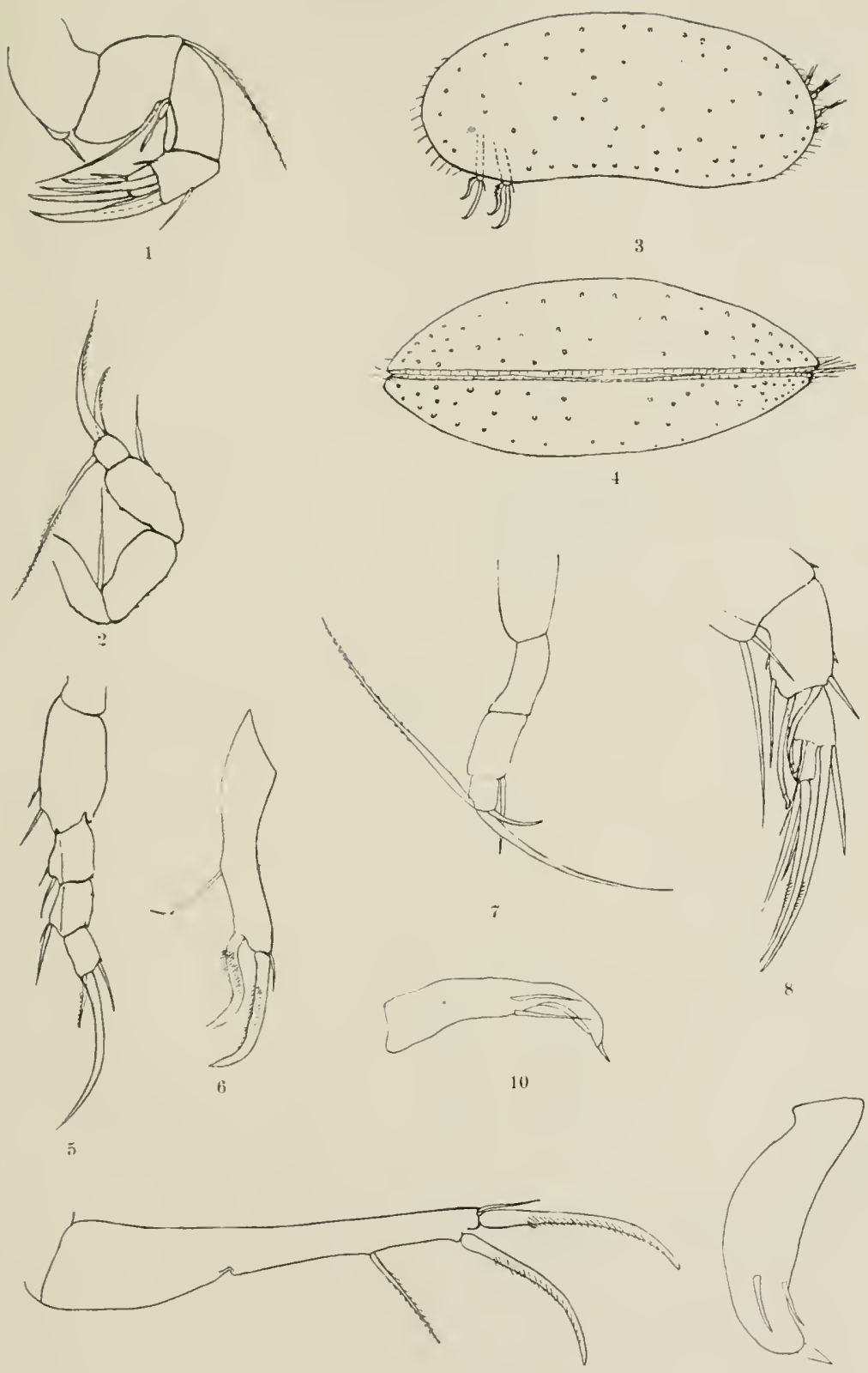

11

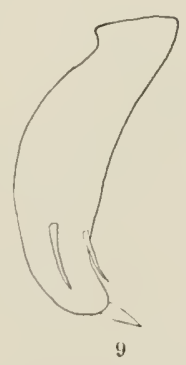


PLATE XlXII.
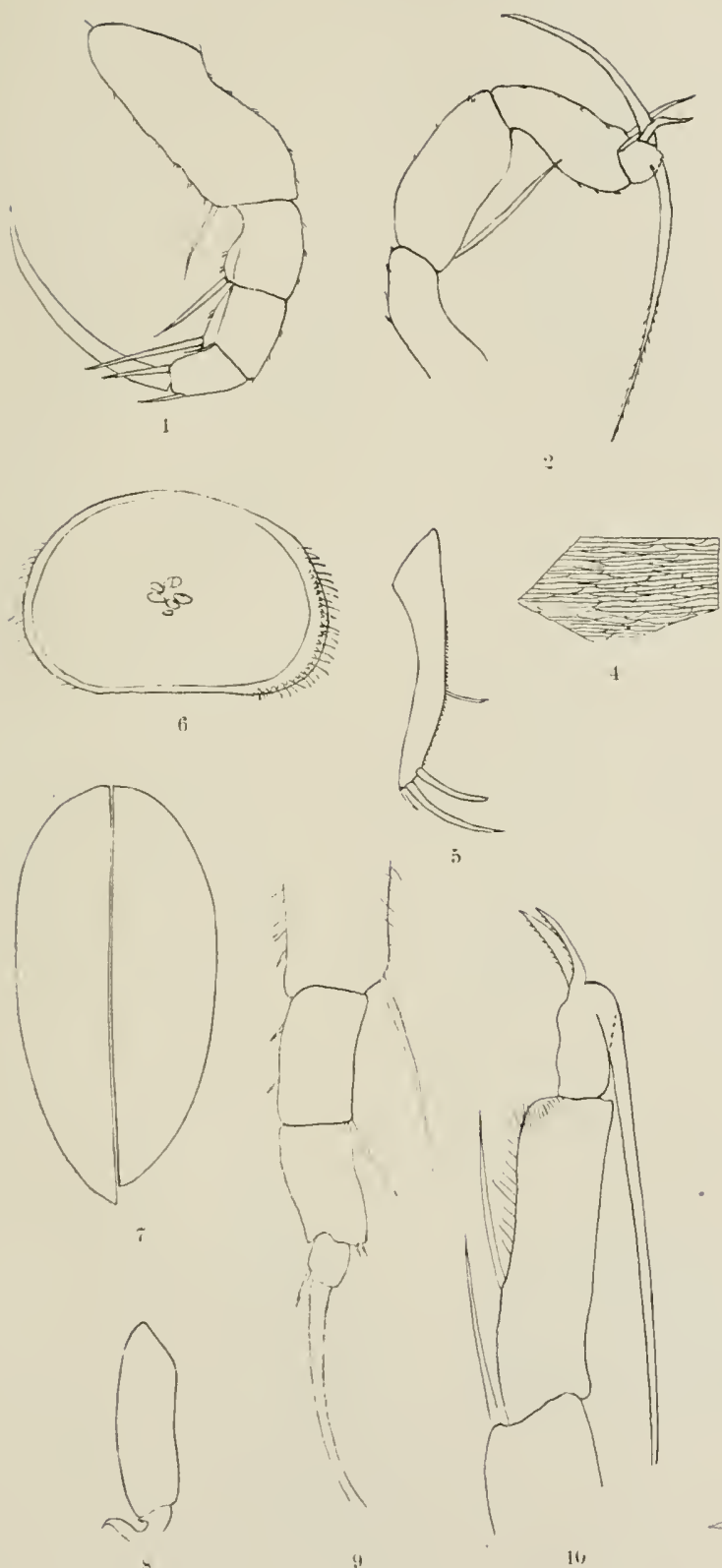

II)

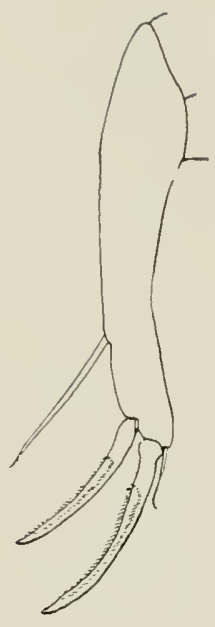

3
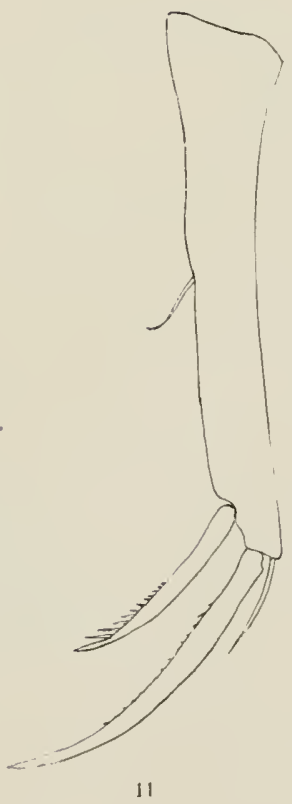
Peate NIRIH.
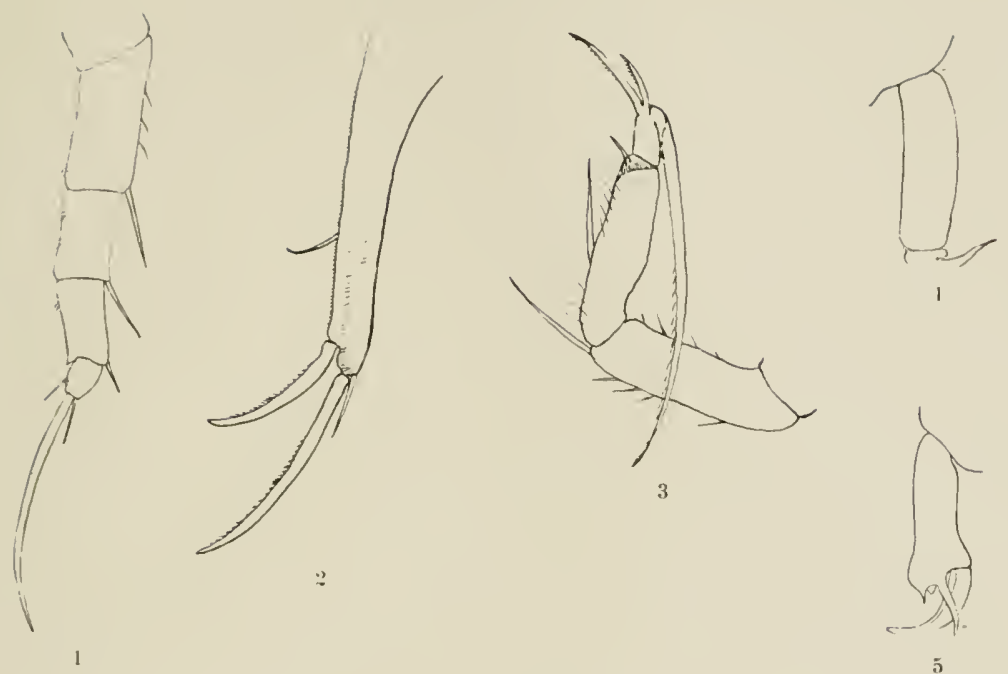

3
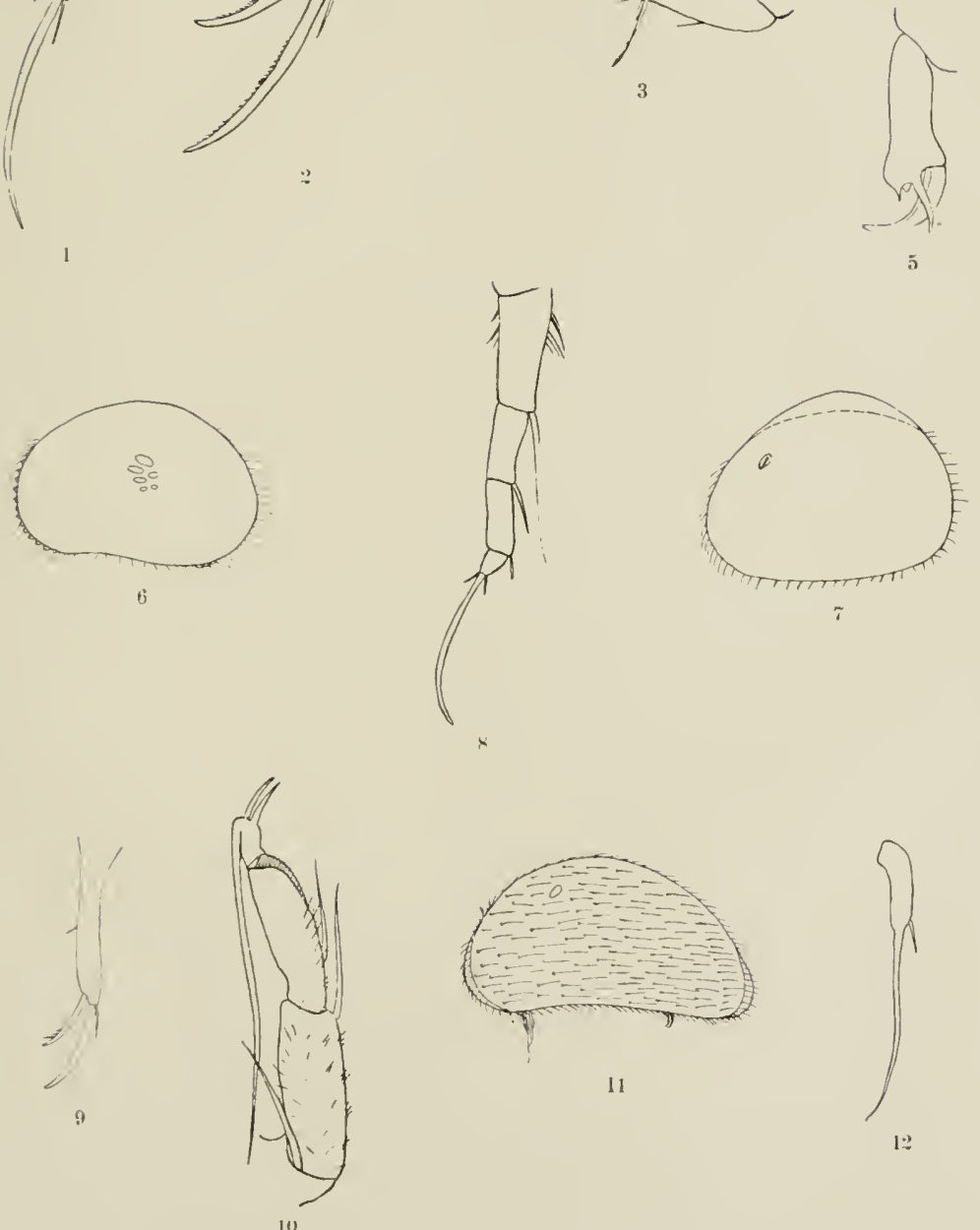


\section{N D E X.}

Algæ, 155, 15\&, 201.

Amalopini, 196.

Amalopis, 196.

Anacharis, 152.

Aquatic caterpillars, $150,155,156$, 157, 163. See Hydrocamplnæ.

Arzama, 164, 165, 166.

Asellus, 156.

Asilidæ, 267.

Atylotus, 232.

Beetles, aquatic, 155, 156, 160, 163.

Bembecidæ, 157, 273.

Bembex spinolæ, $\mathbf{2 7 3 .}$

Berosus, 156, 163.

Bittacomorpha, 190, 200, 201, 206, $228,239,241,278,279$. clavipes, 189, 190.

Black flies, 187. See Simuliidø.

Blepharoceridæ, 184, 187, 188.

Blue earthworm, 157.

Bombyliidæ, 267.

Brachycera, 187, 189.

Braconidæ, 181, 271, 276.

Bugs, aquatic, 155, 156, 161, 163.

Buffalo gnats, 187. See Simuliidæ.

Bull-pout, 221.

Case-flies, 154, 155, 163.

Cataclysta, 165, 166. 174, 175.

Caterpillars, aquatic, $150,155,156$, 157, 163. Seo Hydrocampinse.

Ceratophyllum, 152, 155, 250.

Ceratopogon, 187.

Chalcididæ, 181, 253, 257, 271.

Channel catfish, 221.

Chironomidæ, 155, 184, 187, 189.
Chironomus, 200, 206, 211.

Chrysendeton, 174. claudialis, 174. medicinalis, 174.

Chrysops, 157, 224, 225, 226, 230.

æstuans, $227,279$.

callidus, $\mathbf{2 2 7}$.

excitans, $\mathbf{2 2 6}$.

flavidus, $\mathbf{2 2 7}$.

fugax, $\mathbf{2 2 6}$.

mitis, 226.

montanus, 227.

niger, $2 \mathbf{2 7}$.

obsoletus, 230.

pudicus, $\mathbf{2 2 7}$.

striatus, $\mathbf{2 3 0}$.

univittatu $=\mathbf{2 2 8}$.

vittatus, 228, 279.

Clinowentrus, 276.

niger, 271, $276,278$.

Club rush, 153.

Cnemidotus, 163.

Coccinellid, 157.

Cœnomyia, $\mathbf{2 6 7 .}$

ferruginea, 267.

pallida, $\mathbf{2 6 7 .}$

Coenomyiidæ, 267.

Coleoptera, 158, 163, 164.

Corisidæ, 156.

Cosmocoma maculipes, 172.

Crane-flies, 150, 155, 157, 158, 159, 186. See Tipulidio.

Cremastus, $27 \%$.

hartii, $271, \mathbf{2 7 7}, 278$.

Cryptus eyaneiventris, 178, 270.

Culicidæ, 184, 187, 189. See mosquito: $\mathbf{s .}$

Cyclorhapha, 184, 186. 
Diatoms, 190, 201, 258.

Dicranomyia, 197.

Dicranota, 195, 196, 198.

Diptera, 150, 154, 155, 156, 157, 158 , $161,162,163,164,184,273$. key to families: larvæ and pupæ, 186.

Dixa midges, 187.

Dixidæ, 187, 188.

Dolichopodidæ, 267.

Dragon-flies, 163.

Empididæ, 267.

Ephemerida, 159, 163.

Erioptera, 196, 197.

graphica, 197.

sp. (a), 197, 279.

Eristalis, 239.

False crane-flies, 187.

Fishes, 157, 158.

Flies, 150, 157, 163. See Diptera.

Gammarus, 156.

Geranomyia, 197, 200. canadensis, 200. rostrata, $\mathbf{2 0 0 .}$

Gnats, 187. See Chironomidæ.

Gnophomyia, 196.

Gonomyia, 197.

Green-head, 237.

Gyrinidæ, 155, 156, 163.

Hadronotus, 276.

Haliplids, 155.

Hemiptera, 163. See water-bugs.

Heterocerilæ, 156.

Homophysa, 174, 175.

Horse-flies, 150, 155, 157, 158, 160, 187, 220. See Tabanidœ.

Hydrobatidæ, 155.

Hydrocampa, 157, 165, 166, 167,1 74, $175,179$.

ekthlipsis, 176.

formosalis, 176 .

genuinalis, 176 .

gyralis, $\mathbf{1 7 5}$,

icciusalis, 166, 175, 176.

obliteralis, 166, 168, 174, 175, $176,179,270278$.
Hydiocampa-Continued. peremptalis, 180. proprialis, 176.

Hydrocampinæ, 164, ${ }^{-168,271 . ~ S e 0 ~}$ aquatic caterpillars.

Hymenoptera, 150, 245, 270. parasitic, $157,158,178,181,245$, $250,253,257,264,270$.

Ichneumonidø, $270,277$.

Insects, key to orders, 162. larvæ, 163. nymphs, 163. pupæ, 164.

Isotoma, 156.

Leeches, 155, 156.

Lemna, trisulca, 168, 177, 271.

Lemnaceæ, $2 \tau 1$.

Lepidoptela, 150,163,164, 165,271. key to larvæ, 165. pupæ 166.

Leptidæ, 158, 188, 189, 267. ? larvæ, 26 ?.

?sp. (a), 268.

?sp., (b), 269.

?sp., (c), $2 \mathbf{7 0}$.

Limnobiinæ, 195, 196, 197, 206.

Limnobiini, 197.

Limnophila, 197, 200, 228, 239, 241. luteipennis, 200, 205, 279. sp. (a), 204.

Loxotropa ruficornis, 271.

May-flies, 163. See Ephemerida.

Megilla maculata, 157, 254.

Midges, 187. See Chironomidæ.

Mollusks, 155.

Mosquitoes, 156, 187. See Culicld $\circledast$. Moth-flies, 186. See Psychodidæ.

Muscids, 239.

Nelumbo, 152, 168, 180. lutea, 181.

Nematocera, 186, 188.

Nemotelus, 249, 266. unicolor, 266.

Neuroptera, 163, 164.

Neuropteroids, 155.

Noctuidø, 164, 165, 166. 
Nonagria, 164, 165, 166.

Notonecta, 156.

Nymphøa, 152.

Nymphæella, 166.

dispar, 167.

maculalis, 167.

Oconata, 163.

Edogonium, 152.

Odontomyia, 157, 247, 248, 250, 253, $254,271$.

key to imagos, 255.

larvæ, 254.

binotata, 255, 262.

cincta, 250, 253, 254, 255, 256, $262,264,265,266,271,280$.

extremis, 254, $255,256$.

hieroglyphica, 256, 266.

Intermedia, 255, 256 .

megacephala, 255, 262.

pilimana, 255, 256 .

plebeja. 255, 256.

pubescens, $255 \mathbf{2 5 6}$.

snowi, 256, 266.

vertebrata, 253, 254, 255, 262, $271,28 \%$.

willistoni, 254, 255, 262.

Oligostigma, $174,175$.

Operculate univalves, 157.

Orthorhapha, 150,184, 185, 186, 188 , 267.

key to families : larræ, 186. pupæ, 183.

Pachyrhina, 206.

ferruginea, 210, 216, 217, 218 , 219.

Pangonia, 225, 226.

rasa, 226.

Paraponyx, 165, 166,167, 174,175, 177.

albalis, 173.

allionealls, 173.

obscuralis, 167, 173, 278.

plenilinealis, 173.

stratiotata, 171, 173.

Parasitiea, 270. See Hymenoptera, parasitic.
Pedicia, 196.

Perlid, 159.

Phalacrocera, 196.

Phanurus, 274, 275, 276.

tabani, 276.

tabanivorus, 245, $272,274,280$.

Philhydrus, 163.

Phryganeidæ, 162. See case-flies.

Plecopter'a, 163.

Podura, 156.

Polynẻma natans, 270.

Pompilidæ, 157, 272.

biguttatus, 272.

n. sp?, 272.

philadelphicus, 272.

Poplar, 267.

Potamogeton, 152, 168. nutans, 167, 176, 179, 270.

Proctotrypidæ, 27 1, 274, 275.

Psychodidæ, 184, 186, 188.

Pyralidæ, 164, 165, 166.

Pyramusta, 164, 180. nelumbialis, 165, 166, 180. penitalis, 183.

Rhipidia, 197.

Rhyphidæ, 187, 189.

Rushes, 154, 190, 197, 221, 227, 268.

Sagittaria, 190, 254.

Sand wasps, 157273.

Scirpus, 153.

Sialidæ, 160.

Sialis, 228.

Simuliidæ, 181, 187, 189.

Smicra, 157, 250, 253.

microgaster, 253, 254, 264, 271.

lufolemorata, 250, 253, 271.

Snipe-flies, 188. See Leptidø.

Soldier-flies, 150, 155, 156, 157, 188.

See Stratiomyiidø.

Sparganophilus eiseni, 157.

Spiders, 157, 272.

Spider-wasps, 157. See Pompilidø.

Spring-tails, 156, 161, 163.

Stone-flies, 163.

Straight-seamed flies, 150. See Orthorhapha. 
Stratiomyia, 156, 157, 247, 248, 249, 254.

aplcula, 249.

discalis, 249.

marginalis, $\mathbf{2 4 9}$.

norma, 249, 253, 271, 280.

obesa, $\mathbf{2 4 9}$.

quaternaria, $\mathbf{2 4 9}$.

Stratiomyiidæ, 150, 155, 157, 161, 181, 188, 189, 247.

key to genera, 248.

Stratiomyiinæ, 248.

Surface beetles, 155. See Gyrinidø. bugs, 155.

Symplecta, 196, 197, 199.

punctipennis, 199, 279.

Tabánidœ, 150, 157, 184, 187, 18 ', 199, 220, 230, 235, 241. Seo horseflies.

key to genera: eggs, 225.

larvæ, 226.

рupæ, 226.

Tabanus, 224, 225, 226, 227, 230, 233.

key to species: larvæ, 230. pupæ, 232.

abdominalis, 233.

americanus, $\mathbf{2 4 7}$.

atratus, 199, 224, 231, 232, 239, 241, 242, 272, 275, 280.

atratus group, 224, 225, 231, 232,

bicolor, $23 \mathbf{2}$.

cerastes, $\mathbf{2 3 3 .}$

costalis, 231, 232, $\mathbf{2 3 6}$.

cymatophorus, 233.

epistates, 232.

exul, 233.

fulvulus, $\mathbf{2 3 9}$.

giganteus, $233, \mathbf{2 4 7}$.

lasiophthalmus, 232.

lineola, 23 ।, 231, 232, 235, 279.

lineola group, 224, 225, 231, 232, 239.

nigrescens, 231, 232, 235, $\mathbf{2 3 8 .}$

reinwardtii, $\mathbf{2 3 3}$.

sagax, $\mathbf{2 3 8 .}$

sp. (a), $231,234$.

sp. (b), 231, 241, 280.
Tabanus-Continued.

stygius, 231, 232, 238, 239, 241/ $247,280$.

sulcifrons, $\mathbf{2 3 4 .}$

trimaculatus, $\mathbf{2 3 4 .}$

trispilus, $\mathbf{2 3 2}$.

turbidus, $\mathbf{2 3 3}$

venustus, $\mathbf{2 3 3 .}$

Telenomus, 275, 276.

laricis, $275,276$.

othus $275,276$.

tabani, 275, 276.

Therioplectes, $\mathbf{2 3 2}$.

Thysanura, 162, 163 .

Tipula, 195, 197, 206.

bicornis, 208.

eluta, 208, 210, 215, 217, 218; 279.

Tipulidø, $150,157,158,159,184,186$. $188,190,195,239$.

key to genera: larvæ, 196.

Tipulinæ, 196, 197, 206.

key to species: larvæ, 208.

specific characters: pupæ, 210.

sp. (a), 209, 214.

sp. (b), 209, $215,279$.

sp. (c), 209 .

sp. (d), 209.

sp. (e), 210, 216, 279.

sp. (f), $210, \mathbf{2 1 9}$.

Top-minnows, 155.

Trichoptera, 163, 164. See case-flies.

Tubifex rivulorum, 195.

Univalves, opereulate, 157.

Vallisneria, 152. spiralis, 167.

Veliidæ⿱ 155.

Water beetles, $155,156,160,163$. bugs, 155, 156, 161, 163.

lily, 179.

snails, 221.

striders, 155.

Willow, 153, 154, 190.

Wheat, 209.

Worm, 195.

Xylophagidæ, 267.

Xyluphagus, 267. 